\title{
Time evolution of condensed state of interacting bosons with reduced number fluctuation in a leaky box
}

\author{
Akira Shimizut and Jun-ichi Inoue** \\ Department of Basic Science, University of Tokyo, Komaba, Meguro-ku, Tokyo 153-8902, Japan \\ and Core Research for Evolutional Science and Technology (CREST), JST
}

\begin{abstract}
We study the nonequilibrium time evolution of the Bose-Einstein condensate of interacting bosons confined in a leaky box, when its number fluctuation is initially $(t=0)$ suppressed. We take account of quantum fluctuations of all modes, including $\mathbf{k}=\mathbf{0}$, of the bosons. As the wavefunction of the ground state that has a definite number $N$ of interacting bosons, we use a variational form $|N, \mathbf{y}\rangle$, which is obtained by operating a unitary operator $e^{i G(\mathbf{y})}$ on the number state of free bosons. Using $e^{i G(\mathbf{y})}$, we identify a "natural coordinate" $\hat{b}_{0}$ of the interacting bosons, by which many physical properties can be simply described. The $|N, \mathbf{y}\rangle$ can be represented simply as a number state of $\hat{b}_{0}$, we thus call it the "number state of interacting bosons" (NSIB). To simulate real systems, for which if one fixes $N$ at $t=0 N$ will fluctuate at later times because of a finite probability of exchanging bosons between the box and the environment, we evaluate the time evolution of the reduced density operator $\hat{\rho}(t)$ of the bosons in the box as a function of the leakage flux $J$. We concentrate on the most interesting and nontrivial time stage, i.e., the early time stage for which $J t \ll N$, much earlier than the time when the system approaches the equilibrium state. It is shown that the time evolution can be described very simply as the evolution from a single NSIB at $t<0$, into a classical mixture, with a time dependent distribution, of NSIBs of various values of $N$ at $t>0$. Using $\hat{b}_{0}$, we successfully define the cosine and sine operators for interacting many bosons, by which we can analyze the phase fluctuation in a fully quantum-mechanical manner. We define a new state $|\xi, N, \mathbf{y}\rangle$ called the "number-phase squeezed state of interacting bosons" (NPIB), which is characterized by a complex parameter $\xi$. It is shown that $\hat{\rho}(t)$ for $t>0$ can be rewritten as the phase-randomized mixture (PRM) of NPIBs. Among many possible representations of $\hat{\rho}(t)$, this representation is particularly convenient for analyzing the phase fluctuations and the order parameter. We study the order parameter according to a few typical definitions, as well as their time evolution. It is shown that the off-diagonal long-range order (ODLRO) does not distinguish the NSIB and NPIB. Hence, the order parameter $\Xi$ defined from ODLRO, does not distinguish them, either. On the other hand, the other order parameter $\Psi$, defined as the expectation value of the boson operator $\hat{\psi}$, has different values among these states. In particular, for each element of the PRM of NPIBs, we show that $\Psi$ evolves from zero to a finite value very quickly. Namely, after the leakage of only two or three bosons, each element acquires a full, stable and definite (non-fluctuating) value of $\Psi$.
\end{abstract}

PACS numbers: 03.75.Fi, 05.30.Jp, 05.70.Fh, 05.70.Ln

\section{INTRODUCTION AND SUMMARY}

The Bose-Einstein condensation (BEC) has been observed in various systems [1], including the liquid Helium [2], excitons in photo excited semiconductors [3], and atoms trapped by laser beams [4 6]. Although BEC was originally discussed for free bosons, a condensate of free bosons does not have the superfluidity [1] , hence many-body interactions are essential to interesting behaviors of condensates. The condensed state of interacting bosons in a box of finite volume $V$ is conventionally taken as the state in the Bogoliubov approximation, which we denote by $\left|\alpha_{0}, \mathbf{y}^{c l}\right\rangle^{c l}$ [see Refs. [7],8] and Eq. (20) below]. In this state, the boson number $N$ has finite fluctuation, whose magnitude is $\left\langle\delta N^{2}\right\rangle \gtrsim\langle N\rangle[\mathrm{Eq}$. (29)]. This fluctuation is non-negligible in small systems, such as Helium atoms in a micro bubble [9] and laser-trapped atoms [4] 6], where $\langle N\rangle$ is typically $10^{3}-10^{6}$, and thus $\sqrt{\left\langle\delta N^{2}\right\rangle} /\langle N\rangle=3-0.1 \%$. This means that in such systems if one fixes $N$ with the accuracy better than $3-0.1 \%$, then $\left\langle\delta N^{2}\right\rangle\left\langle\langle N\rangle \text {, thus the state } \mid \alpha_{0}, \mathbf{y}^{c l}\right\rangle^{c l}$ is forbidden, and another state should be realized. In most real systems, there is a finite probability of exchanging bosons between the box and the environment. Hence, if one fixes $N$ at some time, $N$ will fluctuate at later times. Namely, the boson state undergoes a nonequilibrium time evolution when its number fluctuation is initially suppressed. The purpose of this paper is to investigate the time evolution of the interacting bosons in such a case, and to discuss how an order parameter is developed.

We first review and discuss the case where the box is closed and the boson number $N$ is exactly fixed (section II). The ground-state wavefunction of such a case may be obtained by the superposition of Bogoliubov's solution $\left|\alpha_{0}, \mathbf{y}^{c l}\right\rangle^{c l}$ over various values of the phase of $\alpha_{0}$ [Eq. (30)]. The resulting state $\left|N, \mathbf{y}^{c l}\right\rangle^{c l}$ has the same energy as $\left|\alpha_{0}, \mathbf{y}^{c l}\right\rangle^{c l}$ because 
of the degeneracy with respect to the phase of $\alpha_{0}$. (This degeneracy leads to the symmetry breaking.) However, such an expression is not convenient for the analysis of physical properties. To find the ground state in the form that is convenient for analyzing physical properties, we derive an effective Hamiltonian $\hat{H}$ [Eq. (9)], which includes quantum fluctuations of all modes including $\mathbf{k}=0$, from the full Hamiltonian $\hat{H}_{B}$ of interacting bosons. Here, we neglect effects due to spatial inhomogeneity of the boson states in the box, because we are not interested in such effects here, and also because we expect that main physics of the nonequilibrium evolution of our interest would not be affected by such effects. A renormalization constant $Z_{g}$ appears in $\hat{H}$. Although $Z_{g}$ formally diverges [Eq. (7)], the divergence is successfully renormalized, i.e., the final results are independent of $Z_{g}$ and finite. As the ground state of $\hat{H}$, we use a variational form $|N, \mathbf{y}\rangle$, which is similar to that of Girardeau and Arnowitt 10]. This form takes a compact form [Eq. (31)]: it is obtained by operating a simple unitary operator $e^{i G(\mathbf{y})}$ on the $N$-particle state of free bosons, where $G(\mathbf{y})$ is a simple bi-quadratic function of the bare operators [Eq. (32)]. This state has the same energy as $\left|\alpha_{0}, \mathbf{y}^{c l}\right\rangle^{c l}$, or, equivalently, $\left|N, \mathbf{y}^{c l}\right\rangle^{c l}$. (Precisely speaking, they have the same energy density in the macroscopic limit, i.e., when $V \rightarrow \infty$ while keeping the density $n$ finite.)

Using the unitary operator $e^{i G(\mathbf{y})}$, we then identify a "natural coordinate" $\hat{b}_{0}$ [Eq. (47)] of the interacting bosons, by which many physical properties can be simply described (section III). Unlike the quasi-particle operators obtained by the Bogoliubov transformation, $\hat{b}_{0}$ is a nonlinear function of bare operators. Moreover, the Hamiltonian is not diagonal with respect to $\hat{b}_{0}$. Such a nonlinear operator, however, describes the physics very simply. For example, $|N, \mathbf{y}\rangle$ is simply represented as a number state of $\hat{b}_{0}$. We thus call $|N, \mathbf{y}\rangle$ the "number state of interacting bosons" (NSIB). We can also define, through $\hat{b}_{0}$, the cosine and sine operators for interacting bosons [see below]. Moreover, using $\hat{b}_{0}$, we decompose the boson field $\hat{\psi}$ into two parts [Eq. (52)]: one behaves anomalously as $V \rightarrow \infty$ and the other is a normal part. In the decomposition, the non-unity $(|Z|<1)$ renormalization constant $Z$ (which should not be confused with $Z_{g}$ appeared in the Hamiltonian) is correctly obtained. This decomposition formula turns out to be extremely useful in the following analyses.

Using these results, we study the nonequilibrium time evolution of interacting bosons in a leaky box (section IV). The time evolution is induced if one fixes $N$ at some time (thus the boson state at that time is the NSIB), because in most real systems there is a finite probability of exchanging bosons between the box and the environment, hence $N$ will fluctuate at later times. We simulate this situation by the following gedanken experiment: At some time $t<0$ one confines exactly $N$ bosons in a box of volume $V$, and that at $t=0$ a small hole is made in the box, so that a small leakage flux $J$ of the bosons is induced. We concentrate on the analysis of the most interesting and nontrivial time stage; the early time stage for which $J t \ll N$, because it is clear that at later times the system approaches the equilibrium state. We are interested in the reduced density operator of the bosons in the box; $\hat{\rho}(t) \equiv \operatorname{Tr}^{\mathrm{E}}\left[\hat{\rho}^{\text {total }}(t)\right]$, where $\hat{\rho}^{\text {total }}(t)$ denotes the density operator of the total system, and $\operatorname{Tr}^{\mathrm{E}}$ the trace operation over environment's degrees of freedom. We successfully evaluate the time evolution of $\hat{\rho}(t)$ by a method which is equivalent to solving the master equation. Our method gives a physical picture more clearly than the master-equation method. We obtain $\hat{\rho}(t)$ in a general form in which all the details of the box-environment interaction $\hat{H}^{E S}$ have been absorbed in the magnitude of the leakage flux $J$. We show that the time evolution can be described very simply in terms of $\hat{b}_{0}$, as the evolution from a single NSIB at $t<0$, into a classical mixture, with a time dependent distribution, of NSIBs of various values of $N$ at $t>0$ [Eq. (107)].

We then discuss the phase $\phi$ as a variable approximately conjugate to the number $N$ (section $\nabla$ ). To treat the quantum phase properly, we consider the sine and cosine operators, $\sin \phi$ and $\hat{\cos } \phi$. It is generally very difficult to define such operators for interacting many-particle systems. Fortunately, however, in terms of the natural coordinate $\hat{b}_{0}$ we successfully define $\sin \phi$ and $\cos \phi$ for interacting bosons, using which we can analyze the phase property in a fully quantum-mechanical manner. We define a "coherent state of interacting bosons" (CSIB) [Eq. (119)], which, unlike Bogoliubov's ground state $\left|\alpha_{0}, \mathbf{y}^{c l}\right\rangle^{c l}$, exactly has the minimum value of the number-phase uncertainty product [Eq. (128)]. We also define a new state $|\xi, N, \mathbf{y}\rangle[$ Eq. (133)], which we call the "number-phase squeezed state of interacting bosons" (NPIB), which has a smaller phase fluctuation than the CSIB, while keeping the number-phase uncertainty product minimum [Eq. (145)]. We point out that $\hat{\rho}(t)$ for $t>0$ can be represented as the phase-randomized mixture (PRM) of NPIBs. Among many possible representations of $\hat{\rho}(t)$, this representation is particularly convenient for analyzing the phase fluctuations and the order parameter.

We also discuss the action of the measurements (or, their equivalents) of $N$ and of $\phi$ (section VI). The forms of $\hat{\rho}(t)$ after such measurements are discussed. As an example of the phase measurement, we discuss an interference experiment of two condensates which are prepared independently in two boxes. It was already established for noninteracting bosons that the interference pattern is developed for each experimental run (although the interference disappears in the average over many runs) [11] 13]. Using our formula for interacting bosons, we show that the same conclusion is drawn very clearly and naturally for interacting bosons.

We finally consider the order parameter according to a few typical definitions, as well as their time evolution 
(section VII ). We show that the off-diagonal long-range order (ODLRO) does not distinguish NSIB, NPIB and CSIB. Hence, the order parameter $\Xi$ defined from ODLRO [Eq. (166)], does not distinguish them either. On the other hand, the other order parameter $\Psi$, defined as the expectation value of the boson operator $\hat{\psi}$, has different values among these states. In particular, for each element of the PRM of NPIBs, we show that $\Psi$ evolves from zero to a finite value very quickly: After the leakage of only two or three bosons, each element acquires a full, stable and definite (non-fluctuating) value of $\Psi$.

\section{PRELIMINARIES}

\section{A. Phase transitions in finite systems}

We consider the phase transition of interacting bosons confined in a large, but finite box of volume $V$. Phase transitions are usually discussed in systems with an infinite volume (or, the $V \rightarrow \infty$ limit is taken at the end of the calculation), because infinite degrees of freedom are necessary in the relevant energy scale for strict transitions [14]. In such a case, we must select a single physical Hilbert space among many possibilities, which selection corresponds to a strict phase transition. However, phase transitions do occur even in systems of finite $V$ in the sense that a single phase lasts longer than the time of observation if its linear dimension exceeds the correlation length at the temperature of interest [14]. Hence, it is physically interesting and important to explore phase transitions in finite systems. Because of the finiteness of $V$ (and the fact that the interaction potential $U$ is well-behaved), the von Neumann's uniqueness theorem can be applied. This allows us to develop a theory in a unique Hilbert space. However, since $V$ is large, some quantities, which become anomalous in the limit of $V \rightarrow \infty$ due to a strict phase transition, behave quasi anomalously. In later sections, we shall identify such a quasi anomalous operator, and discuss how an order parameter is developed.

\section{B. Effective Hamiltonian}

We start from the standard Hamiltonian for interacting bosons confined in a large, but finite box of volume $V$ :

$$
\hat{H}_{B}=\int_{V} d^{3} r \hat{\psi}^{\dagger}(\mathbf{r})\left(-\frac{\hbar^{2}}{2 m} \nabla^{2}\right) \hat{\psi}(\mathbf{r})+\frac{1}{2} \int_{V} d^{3} r \int_{V} d^{3} r^{\prime} \hat{\psi}^{\dagger}(\mathbf{r}) \hat{\psi}^{\dagger}\left(\mathbf{r}^{\prime}\right) U\left(\mathbf{r}-\mathbf{r}^{\prime}\right) \hat{\psi}\left(\mathbf{r}^{\prime}\right) \hat{\psi}(\mathbf{r}) .
$$

Here, we neglect a confining potential of the box because in the present work we are not interested in its effects such as the spatial inhomogeneity of the boson states in the box, and also because we expect that main physics of the nonequilibrium evolution of our interest would not be affected by such effects. (Mathematically, our model under the periodic boundary condition assumes bosons confined in a three-dimensional torus.) The $\mathbf{r}$ dependence of the boson field $\hat{\psi}(\mathbf{r})$ (in the Schrödinger picture) can be expanded in terms of plane waves as

$$
\hat{\psi}(\mathbf{r})=\frac{1}{\sqrt{V}} \hat{a}_{0}+\sum_{\mathbf{k} \neq \mathbf{0}} \frac{e^{i \mathbf{k} \cdot \mathbf{r}}}{\sqrt{V}} \hat{a}_{\mathbf{k}},
$$

where $\hat{a}_{\mathbf{p}}$ and $\hat{a}_{\mathbf{p}}^{\dagger}$ are called creation and annihilation operators, respectively, of bare bosons. The total number of bosons is given by

$$
\hat{N} \equiv \int_{V} d^{3} r \hat{\psi}^{\dagger}(r) \hat{\psi}(r)=\sum_{\mathbf{k}} \hat{a}_{\mathbf{k}}^{\dagger} \hat{a}_{\mathbf{k}}
$$

We assume zero temperature, and consider the case where the interaction is weak and repulsive [Eq. (8) below], and where the boson density $n$ is finite (hence, since $V$ is large, $N \gg 1$ ):

$$
n \equiv\langle N\rangle / V>0
$$

In such a case, BEC occurs and typical matrix elements of $\hat{a}_{0}, \hat{a}_{0}^{\dagger}$ and $\hat{N}$ are huge, whereas those of $\hat{a}_{\mathbf{k}}$ and $\hat{a}_{\mathbf{k}}^{\dagger}$ (with $\mathbf{k} \neq \mathbf{0}$ ) are small. Taking up to the second order terms in these small quantities, and using the identity, $\hat{N}=\hat{a}_{0}^{\dagger} \hat{a}_{0}+\sum_{\mathbf{k} \neq \mathbf{0}} \hat{a}_{\mathbf{k}}^{\dagger} \hat{a}_{\mathbf{k}}$, we obtain the effective Hamiltonian $\hat{H}$ in the following form. 


$$
\hat{H}=g\left(1+Z_{g}\right) \frac{\hat{N}^{2}}{2 V}-g\left(1+Z_{g}\right) \frac{1}{2 V} \hat{a}_{0}^{\dagger} \hat{a}_{0}+\sum_{\mathbf{k} \neq \mathbf{0}}\left(\epsilon_{k}^{(0)}+g \frac{\hat{N}}{V}\right) \hat{a}_{\mathbf{k}}^{\dagger} \hat{a}_{\mathbf{k}}+\left[\frac{g}{2 V} \hat{a}_{0} \hat{a}_{0} \sum_{\mathbf{k} \neq \mathbf{0}} \hat{a}_{\mathbf{k}}^{\dagger} \hat{a}_{-\mathbf{k}}^{\dagger}+\text { h.c. }\right] \text {. }
$$

Here, $\epsilon_{k}^{(0)}$ denotes the free-particle energy, $\epsilon_{k}^{(0)} \equiv \hbar^{2} k^{2} / 2 m$, and $g$ is an effective interaction constant defined by

$$
g \equiv \frac{4 \pi \hbar^{2} a}{m} .
$$

Here, $a$ is the scattering length, and $Z_{g}$ is the first-order "renormalization constant" for the scattering amplitude [15];

$$
Z_{g} \equiv \frac{g}{2 V} \sum_{\mathbf{k} \neq \mathbf{0}} \frac{1}{\epsilon_{k}^{(0)}}
$$

The formal divergence of the sum in Eq. (7) does not matter because the final results are independent of $Z_{g}$ [15], hence the renormalization is successful. We have assumed that

$$
0<n a^{3} \ll 1,
$$

under which the approximation $\hat{H}_{B} \approx \hat{H}$ is good. We have confirmed by explicit calculations that the term $-\{g(1+$ $\left.\left.Z_{g}\right) / 2 V\right\} \hat{a}_{0}^{\dagger} \hat{a}_{0}$ in Eq. (5) gives only negligible contributions in the following analysis. We thus drop it henceforth:

$$
\hat{H}=g\left(1+Z_{g}\right) \frac{\hat{N}^{2}}{2 V}+\sum_{\mathbf{k} \neq \mathbf{0}}\left(\epsilon_{k}^{(0)}+g \frac{\hat{N}}{V}\right) \hat{a}_{\mathbf{k}}^{\dagger} \hat{a}_{\mathbf{k}}+\left[\frac{g}{2 V} \hat{a}_{0} \hat{a}_{0} \sum_{\mathbf{k} \neq \mathbf{0}} \hat{a}_{\mathbf{k}}^{\dagger} \hat{a}_{-\mathbf{k}}^{\dagger}+\text { h.c. }\right] .
$$

Since this $\hat{H}$ commutes with $\hat{N}$, we can in principle find its eigenstates for which $N$ is exactly fixed. In each subspace of fixed $N, \hat{H}$ is equivalent to

$$
\hat{H}(N) \equiv \frac{1}{2} g\left(1+Z_{g}\right) n^{2} V+\sum_{\mathbf{k} \neq \mathbf{0}} \epsilon_{k}^{\prime} \hat{a}_{\mathbf{k}}^{\dagger} \hat{a}_{\mathbf{k}}+\left[\frac{g}{2 V} \hat{a}_{0} \hat{a}_{0} \sum_{\mathbf{k} \neq \mathbf{0}} \hat{a}_{\mathbf{k}}^{\dagger} \hat{a}_{-\mathbf{k}}^{\dagger}+\text { h.c. }\right],
$$

where

$$
\epsilon_{k}^{\prime} \equiv \epsilon_{k}^{(0)}+g n
$$

Note that if we regard $\hat{a}_{0}$ in $\hat{H}(N)$ as a classical complex number [16]

$$
\hat{a}_{0} \rightarrow e^{i \phi} \sqrt{N_{0}} \equiv \alpha_{0}
$$

and $\hat{a}_{0}^{\dagger}$ as $\alpha_{0}^{*}$, we would then obtain the "semiclassical" Hamiltonian $\hat{H}^{c l}$ as

$$
\hat{H}^{c l}=\frac{1}{2} g\left(1+Z_{g}\right) n^{2} V+\sum_{\mathbf{k} \neq \mathbf{0}} \epsilon_{k}^{\prime} \hat{a}_{\mathbf{k}}^{\dagger} \hat{a}_{\mathbf{k}}+\left(\frac{1}{2} g n e^{2 i \phi} \sum_{\mathbf{k} \neq \mathbf{0}} \hat{a}_{\mathbf{k}}^{\dagger} \hat{a}_{-\mathbf{k}}^{\dagger}+\text { h.c. }\right),
$$

where we have replaced $N_{0}$ with $N$ in the last parenthesis because the replacement just gives correction which is of higher order in $g$. This Hamiltonian can be diagonalized exactly (See, e.g., Ref. [15] in which $\phi=0$ ). We shall utilize this fact later to find the ground state of $\hat{H}$.

\section{Known results for non-interacting bosons}

When $g=0$, the ground state of free bosons whose number $N$ is fixed, is simply a number state,

$$
|N\rangle \equiv \frac{1}{\sqrt{N !}}\left(\hat{a}_{0}^{\dagger}\right)^{N}|0\rangle
$$


where $|0\rangle$ denotes the vacuum of the bare operators;

$$
\hat{a}_{\mathbf{k}}|0\rangle=0 \text { for all } \mathbf{k} \text {. }
$$

The energy of $|N\rangle$,

$$
E_{N}=N \epsilon_{0}^{(0)}=0,
$$

is degenerate with respect to $N$. Hence, any superposition of $|N\rangle$ is also a ground state. For example, the coherent state

$$
|\alpha\rangle \equiv e^{-|\alpha|^{2} / 2} \sum_{N=0}^{\infty} \frac{\alpha^{N}}{\sqrt{N !}}|N\rangle=e^{-|\alpha|^{2} / 2} e^{\alpha \hat{a}_{0}^{\dagger}}|0\rangle,
$$

where $\alpha \equiv e^{i \phi} \sqrt{N}$, is also a ground state that has the same expectation value of $\hat{N}$ as $|N\rangle$. On the other hand, $|\alpha\rangle$ has a finite fluctuation of $N$,

$$
\left\langle\delta N^{2}\right\rangle \equiv\left\langle(\hat{N}-\langle\hat{N}\rangle)^{2}\right\rangle=|\alpha|^{2}=\langle N\rangle,
$$

whereas $|N\rangle$ has a definite $N$. The inverse transformation from $|\alpha\rangle$ to $|N\rangle$ can be accomplished as

$$
|N\rangle=\int_{-\pi}^{\pi} \frac{d \phi}{2 \pi}|\alpha\rangle .
$$

\section{Known results for $\hat{H}^{c l}$}

Neither $|N\rangle$ nor $|\alpha\rangle$ is an eigenstate when $g>0$. If we can regard $\hat{a}_{0}$ and $\hat{a}_{0}^{\dagger}$ as classical numbers $\alpha_{0}\left(=e^{i \phi} \sqrt{N_{0}}\right)$ and $\alpha_{0}^{*}$, respectively, we can use $\hat{H}^{c l}$ as the Hamiltonian, and its ground state was given by Bogoliubov as [1, 4,8

$$
\left|\alpha_{0}, \mathbf{y}^{c l}\right\rangle^{c l} \equiv \exp \left[\left(\alpha_{0} \hat{a}_{0}^{\dagger}-\frac{1}{2} \sum_{\mathbf{q} \neq \mathbf{0}} y_{q}^{c l *} \hat{a}_{\mathbf{q}}^{\dagger} \hat{a}_{-\mathbf{q}}^{\dagger}\right)-\text { h.c. }\right]|0\rangle,
$$

where

$$
\begin{aligned}
y_{q}^{c l} & =\left|y_{q}^{c l}\right| e^{-2 i \phi}, \\
\cosh \left|y_{q}^{c l}\right| & =\sqrt{\frac{\epsilon_{q}+\epsilon_{q}^{(0)}+g n}{2 \epsilon_{q}}}, \\
\sinh \left|y_{q}^{c l}\right| & =\frac{g n}{\sqrt{2 \epsilon_{q}\left(\epsilon_{q}+\epsilon_{q}^{(0)}+g n\right)}} .
\end{aligned}
$$

Here, $\epsilon_{q}$ is the quasi-particle energy,

$$
\epsilon_{q} \equiv \sqrt{\epsilon_{q}^{(0)}\left(\epsilon_{q}^{(0)}+2 g n\right)}
$$

whose dispersion is linear for $\epsilon_{q}^{(0)} \ll g n$;

$$
\epsilon_{q} \approx \sqrt{\frac{g n}{m}}|q|
$$

The ground state energy is calculated as 15

$$
\begin{aligned}
E_{\alpha, \mathbf{y}^{c l}}^{c l} & =\frac{1}{2} g n N\left(1+\frac{128}{15} \sqrt{\frac{n a^{3}}{\pi}}\right) \\
& =\frac{1}{2} g n N+O\left(g^{2.5}\right) .
\end{aligned}
$$


The absence of the $O\left(g^{2}\right)$ term in $E_{\alpha, \mathbf{y}^{c l}}^{c l}$ means that the large (formally divergent because $Z_{g} \rightarrow \infty$ ) positive energy $g Z_{g} n N / 2$ of $\hat{H}^{c l}$ is canceled by a large negative term arising from the pair correlations. The expectation value and variance of $\hat{N}$ for $\left|\alpha_{0}, \mathbf{y}^{c l}\right\rangle^{c l}$ are evaluated as

$$
\begin{aligned}
\langle N\rangle^{c l} & =N_{0}+\sum_{\mathbf{q} \neq \mathbf{0}}\left(\sinh \left|y_{q}^{c l}\right|\right)^{2}, \\
\left\langle\delta N^{2}\right\rangle^{c l} & =\langle N\rangle^{c l}+\sum_{\mathbf{q} \neq \mathbf{0}}\left(\sinh \left|y_{q}^{c l}\right|\right)^{4} .
\end{aligned}
$$

\section{E. Ground state of a fixed number of bosons}

In analogy to Eq. (19), it is possible to construct an approximate ground state of $\hat{H}(N)$ from $\left|\alpha_{0}, \mathbf{y}^{c l}\right\rangle^{c l}$ as

$$
\left|N, \mathbf{y}^{c l}\right\rangle^{c l} \equiv \int_{-\pi}^{\pi} \frac{d \phi}{2 \pi}\left|\alpha_{0}, \mathbf{y}^{c l}\right\rangle^{c l}
$$

where $N \equiv\langle N\rangle^{c l}$, Eq. (28). We can obtain an explicit form of $\left|N, \mathbf{y}^{c l}\right\rangle^{c l}$ in the form of an infinite series expansion, by inserting Eq. (20) into the right-hand side (rhs) of Eq. (30), expanding the exponential function, and performing the $\phi$ integral. However, such an expression is not convenient for the analysis of physical properties. Note that in many-particle physics, it is generally difficult to evaluate physical properties even if the wavefunction is known. It is therefore essential to find the ground state in the form that is convenient for analyzing physical properties.

Several formulations were developed for the condensation of interacting bosons with a fixed $N$. Lifshitz and Pitaevskii [15] developed a formal discussion for the case of fixed $N$. However, they did not treat $\hat{a}_{0}$ as an operator, hence $\hat{N}$ was not conserved. For example, $\hat{b}_{p}^{\dagger}|m, N\rangle$ (in their notations) did not have exactly $N+1$ bosons. To treat interacting bosons with fixed $N$ more accurately, one has to include quantum fluctuations of all modes (including $\mathbf{k}=\mathbf{0}$ ), by treating $\hat{a}_{0}$ as an operator. Such treatment was developed, for example, by Girardeau and Arnowitt [10], Gardiner [17], and Castin and Dum [18]. A variational form was proposed in Ref. [10] for the wavefunction of the ground state. The variational form takes account of four-particle correlations in an elaborate manner, and is normalized exactly. On the other hand, in Refs. [17] and [18] no explicit form was derived for the ground-state wavefunction. [These references are more interested in excited states and the spatially inhomogeneous case, rather than the ground-state wavefunction.] We here use a variational form, which is similar to that of Ref. [10], as the ground state of $\hat{H}(N)$;

$$
|N, \mathbf{y}\rangle \equiv e^{i G(\mathbf{y})} \frac{1}{\sqrt{N !}}\left(\hat{a}_{0}^{\dagger}\right)^{N}|0\rangle
$$

Here, $\hat{G}(\mathbf{y})$ is the hermite operator defined by

$$
\hat{G}(\mathbf{y}) \equiv \frac{-i}{2 n V} \hat{a}_{0}^{\dagger} \hat{a}_{0}^{\dagger} \sum_{\mathbf{q} \neq \mathbf{0}}\left|y_{q}^{c l}\right| \hat{a}_{\mathbf{q}} \hat{a}_{-\mathbf{q}}+\text { h.c. }
$$

where $\mathbf{y} \equiv\left\{y_{q}\right\}$ are a set of variational parameters, which are taken as

$$
y_{q}=\left|y_{q}^{c l}\right| .
$$

Using the well-known formula for arbitrary operators $\hat{A}$ and $\hat{B}$,

$$
e^{\hat{A}} \hat{B} e^{-\hat{A}}=\hat{B}+[\hat{A}, \hat{B}]+\frac{1}{2 !}[\hat{A},[\hat{A}, \hat{B}]]+\cdots,
$$

we find from Eqs. (31)-(32) that

$$
E_{N, \mathbf{y}} \equiv\langle N, \mathbf{y}|\hat{H}| N, \mathbf{y}\rangle=\frac{1}{2} g n N+o\left(g^{2}\right) .
$$

where $o\left(g^{2}\right)$ denotes terms which tend to zero as $g \rightarrow 0$, faster than $g^{2}$. This demonstrates that the large (formally divergent because $Z_{g} \rightarrow \infty$ ) positive energy $g Z_{g} n N / 2$ in $\hat{H}(N)$ is canceled by a large negative term arising from the 
four-particle correlations of the state of Eq. (31). Moreover, we can also show that in the macroscopic limit $(V \rightarrow \infty$ while keeping $n$ constant), $E_{N, \mathbf{y}}$ becomes as low as $E_{\alpha, \mathbf{y}^{c l}}^{c l}$ of Eq. (26). Therefore, the form of Eq. (31) is a good approximation to the ground state. Note that $|N, \mathbf{y}\rangle$ is an eigenstate of $\hat{N}$;

$$
\hat{N}|N, \mathbf{y}\rangle=N|N, \mathbf{y}\rangle,
$$

hence $|N, \mathbf{y}\rangle$ is an (approximate) groundstate of $\hat{H}$;

$$
\hat{H}|N, \mathbf{y}\rangle=\hat{H}(N)|N, \mathbf{y}\rangle .
$$

Note also that $|N, \mathbf{y}\rangle$ is exactly normalized to unity because $e^{i G(\mathbf{y})}$ in Eq. (31) is a unitary transformation (although it becomes non-unitary in the limit of $V \rightarrow \infty$ ).

We should make a remark here. In the case of $\left|\alpha, \mathbf{y}^{c l}\right\rangle^{c l}$ discussed in subsection IID, $E_{\alpha, \mathbf{y}^{c l}}^{c l}$ becomes low enough only for the specific choice of the phase of $y_{q}^{c l}[$ Eq. [21] ]. This phase relation is sometimes called the "phase locking" [7]. From this viewpoint, it is sometimes argued [7, 19] that "having a definite phase" and the "phase locking" are necessary to achieve a low energy. However, such a statement is rather misleading: In our case, the phase locking corresponds to the fact that $y_{q}$ 's are real and positive. On the other hand, our ground state $|N, \mathbf{y}\rangle$ has no fluctuation in $N$, hence has no definite phase [20] because of the number-phase uncertainty relation,

$$
\left\langle\delta N^{2}\right\rangle\left\langle\delta \phi^{2}\right\rangle \gtrsim 1 / 4
$$

Nevertheless, the energy of $|N, \mathbf{y}\rangle$ is as low as $E_{\alpha, \mathbf{y}^{c l}}^{c l}$. That is, "having a definite phase" is not necessary to achieve the ground-state energy, and thus the term "phase locking" should be taken carefully.

\section{F. Ground state of $N-\Delta N$ bosons}

The ground state of $N-\Delta N$ bosons is given by Eqs. (31) and (32) in which $N$ is all replaced with $N-\Delta N$. However, we are interested in the case where [see section IV]

$$
|\Delta N| \ll N .
$$

In this case, $\hat{G}(\mathbf{y})$ and $\mathbf{y}$ (which are functions of the density of bosons) of $N-\Delta N$ bosons are almost identical to those of $N$ bosons because $(N-\Delta N) / V \approx N / V=n$. Therefore, we can simplify the calculation by using the same $\hat{G}(\mathbf{y})$ and $\mathbf{y}$ for all $\Delta N$. That is, we take

$$
|N-\Delta N, \mathbf{y}\rangle=e^{i G(\mathbf{y})} \frac{1}{\sqrt{(N-\Delta N) !}}\left(\hat{a}_{0}^{\dagger}\right)^{N-\Delta N}|0\rangle,
$$

where $\hat{G}(\mathbf{y})$ and $\mathbf{y}$ are those of $N$ bosons. Despite the approximation, this state is exactly normalized and has exactly $N-\Delta N$ bosons;

$$
\hat{N}|N-\Delta N, \mathbf{y}\rangle=(N-\Delta N)|N-\Delta N, \mathbf{y}\rangle .
$$

\section{NATURAL COORDINATE}

\section{A. Nonlinear Bogoliubov transformation}

Since we assume that $V$ is finite, $e^{i G(\mathbf{y})}$ is a unitary operator (which, however, becomes non-unitary in the limit of $V \rightarrow \infty)$. Utilizing this fact, we define new boson operators $\hat{b}_{\mathbf{k}}$ by

$$
\hat{b}_{\mathbf{k}} \equiv e^{i \hat{G}(\mathbf{y})} \hat{a}_{\mathbf{k}} e^{-i \hat{G}(\mathbf{y})} .
$$

This operator satisfies the same commutation relations as $\hat{a}_{\mathbf{k}}$;

$$
\left[\hat{b}_{\mathbf{p}}, \hat{b}_{\mathbf{q}}^{\dagger}\right]=\delta_{\mathbf{p}, \mathbf{q}}, \quad\left[\hat{b}_{\mathbf{p}}, \hat{b}_{\mathbf{q}}\right]=\left[\hat{b}_{\mathbf{p}}^{\dagger}, \hat{b}_{\mathbf{q}}^{\dagger}\right]=0 .
$$


Note that these relations are exact, in contrast to the operator $b_{\mathbf{k}}(\mathbf{k} \neq \mathbf{0})$ of Ref. [17]. Owing to the exact commutation relations, we can define the vacuum of $\hat{b}_{\mathrm{k}}$ 's by

$$
\hat{b}_{\mathbf{k}}|0, \mathbf{y}\rangle=0 \quad \text { for all } \mathbf{k} .
$$

From Eqs. (15), (32) and (42), we have 21]

$$
|0, \mathbf{y}\rangle=e^{i \hat{G}(\mathbf{y})}|0\rangle .
$$

The transformation (42) somewhat resembles the Bogoliubov transformation which diagonalizes $\hat{H}^{c l}$ [15]. However, in contrast to Bogoliubov's quasi-particles (whose total number differs from $\hat{N}$ as an operator), the total number operator of $\hat{b}_{\mathbf{k}}$ 's is identical to that of $\hat{a}_{\mathbf{k}}$ 's because $[\hat{N}, \hat{G}(\mathbf{y})]=0$;

$$
\sum_{\mathbf{k}} \hat{b}_{\mathbf{k}}^{\dagger} \hat{b}_{\mathbf{k}}=e^{i \hat{G}(\mathbf{y})} \hat{N} e^{-i \hat{G}(\mathbf{y})}=\hat{N} .
$$

This property is very useful in the following analyses. On the other hand, the transformation (42) is much more complicated than the Bogoliubov transformation: The latter is a linear transformation connecting the bare operators with quasi-particle operators, whereas the former is a nonlinear transformation between the bare operators and the new boson operators. For example, $\hat{b}_{0}$ defined by

$$
\hat{b}_{0} \equiv e^{i \hat{G}(\mathbf{y})} \hat{a}_{0} e^{-i \hat{G}(\mathbf{y})}
$$

is a rather complicated, nonlinear function of $\hat{a}_{\mathbf{k}}$ 's and $\hat{a}_{\mathbf{k}}^{\dagger}$ 's (of various k's including $\mathbf{k}=\mathbf{0}$ ), as can be seen using Eq. (34). Such a nonlinear operator $\hat{b}_{0}$, however, describes the physics quite simply. Namely, we show that $\hat{b}_{0}$ (and $\hat{b}_{0}^{\dagger}$ ) is a "natural coordinate" [22 of interacting bosons in the sense that many physical properties can be simply described. (It is crucial to find such a coordinate for the analysis of many-particle systems, because generally the knowledge of the wavefunction is not sufficient to perform the analysis.) For example, from Eqs. (31), (42) and (44), we find that in terms of $\hat{b}_{0}$ the ground state $|N, \mathbf{y}\rangle$ is simply a number state;

$$
|N, \mathbf{y}\rangle=\frac{1}{\sqrt{N !}}\left(\hat{b}_{0}^{\dagger}\right)^{N}|0, \mathbf{y}\rangle
$$

In particular,

$$
\hat{b}_{0}|N, \mathbf{y}\rangle=\sqrt{N}|N-1, \mathbf{y}\rangle .
$$

Since $\hat{b}_{\mathbf{k}}$ 's of $\mathbf{k} \neq \mathbf{0}$ commute with $\hat{b}_{0}$, we also find that

$$
\hat{b}_{\mathbf{k}}|N, \mathbf{y}\rangle=0 \quad \text { for all } \mathbf{k} \neq \mathbf{0}
$$

Therefore, in terms of the new boson operators the ground state of the interacting bosons can be simply viewed as a single-mode $(\mathbf{k}=\mathbf{0})$ number state. Note, however, that this does not mean that $\hat{H}$ were bilinear and diagonal with respect to $\hat{b}_{0}$. In fact, if it were the case then the energy $E_{N, \mathbf{y}}$ would be linear in $N$, in contradiction to Eq. (35) which shows $E_{N, \mathbf{y}} \propto N^{2}$ (recall that $n=N / V$ ).

The usefulness of $\hat{b}_{0}$ are strongly suggested by Eqs. (48) and (50). We will show in the following discussions that this is indeed the case.

\section{B. Decomposition of $\hat{\psi}$}

Some matrix elements of $\hat{b}_{0}$ become anomalously large, among the ground (and excited) states of different $N$. For example,

$$
\left\langle N-1, \mathbf{y}\left|\hat{b}_{0}\right| N, \mathbf{y}\right\rangle=\sqrt{N}=\sqrt{n V} .
$$

This indicates that in the $V \rightarrow \infty$ limit (while keeping the density $n$ finite) $\hat{b}_{0}$ does not remain an annihilation operator of the physical Hilbert space, signaling that a strict phase transition should occur as $V \rightarrow \infty$. Since this anomaly 
should have important effects even for a finite $V$, it is appropriate to separate $\hat{b}_{0}$ from the other terms of $\hat{\psi}$. That is, we decompose the boson field in a finite system as (cf. Eq. (2))

$$
\hat{\psi}=\frac{Z^{1 / 2}}{\sqrt{V}} \hat{b}_{0}+\hat{\psi}^{\prime}
$$

where $Z$ is a complex renormalization constant. Since we have specified nothing about $\hat{\psi}^{\prime}$ at this stage, this decomposition is always possible and $Z$ is arbitrary. Following Ref. [15], we define the "wavefunction of the condensate" $\Xi$ by

$$
\Xi \equiv\langle N-1, \mathbf{y}|\hat{\psi}| N, \mathbf{y}\rangle
$$

Since $\Xi$ is independent of $\mathbf{r}$ (because both $|N-1, \mathbf{y}\rangle$ and $|N, \mathbf{y}\rangle$ have the translational symmetry), we can take $Z$ as

$$
\Xi=Z^{1 / 2}\left\langle N-1, \mathbf{y}\left|\hat{b}_{0}\right| N, \mathbf{y}\right\rangle / \sqrt{V}
$$

That is, from Eq. (48),

$$
Z^{1 / 2}=\Xi / \sqrt{n}
$$

Then, by taking the matrix element of Eq. (52) between $|N-\Delta N, \mathbf{y}\rangle$ and $|N, \mathbf{y}\rangle$, we find

$$
\left\langle N-\Delta N, \mathbf{y}\left|\hat{\psi}^{\prime}\right| N, \mathbf{y}\right\rangle=0 \quad \text { for }{ }^{\forall} \Delta N(|\Delta N| \ll N) .
$$

We now define two number operators by

$$
\begin{aligned}
& \hat{N}^{\prime} \equiv \int d^{3} r \hat{\psi}^{\prime \dagger}(r) \hat{\psi}^{\prime}(r), \\
& \hat{N}_{0} \equiv \hat{N}-\hat{N}^{\prime} .
\end{aligned}
$$

Then, from Eqs. (48), (52), (55), and (56), we find

$$
\langle N, \mathbf{y}|\hat{N}| N, \mathbf{y}\rangle=V|\Xi|^{2}+\left\langle N, \mathbf{y}\left|\hat{N}^{\prime}\right| N, \mathbf{y}\right\rangle
$$

Hence, from Eq. (58),

$$
\left\langle N, \mathbf{y}\left|\hat{N}_{0}\right| N, \mathbf{y}\right\rangle=V|\Xi|^{2},
$$

which may be interpreted as the "number of condensate particles" [15]. That is, in agreement with the standard result $15,|\Xi|^{2}$ is the density of the condensate particles;

$$
|\Xi|^{2}=\left\langle N_{0}\right\rangle / V \equiv n_{0},
$$

where we have denoted the expectation value simply by $\langle\cdots\rangle$. We can therefore write $\Xi$ as

$$
\Xi=\sqrt{n_{0}} e^{i \varphi} \text {. }
$$

We thus find the formula for the decomposition of $\hat{\psi}$ as

$$
\hat{\psi}=e^{i \varphi} \sqrt{\frac{n_{0}}{n V}} \hat{b}_{0}+\hat{\psi}^{\prime}
$$

which is extremely useful in the following analysis. Note that we have obtained the finite renormalization;

$$
|Z|=n_{0} / n<1 .
$$




\section{Relation to the previous work}

Lifshitz and Pitaevskii 15 introduced an operator $\hat{\Xi}$ that transforms an eigenstate with $N$ bosons into the corresponding eigenstate with $N-1$ bosons, without giving an explicit form of $\hat{\Xi}$. (They defined $\hat{\Xi}$ through its matrix elements between eigenstates with different values of $N$. However, as mentioned in section IIE, they did not give the forms of the eigenstates of fixed $N$.) They decomposed $\hat{\psi}$ as (Eq. (26.4) of Ref. [15])

$$
\hat{\psi}=\hat{\Xi}+\hat{\psi}^{\prime} \text {. }
$$

In the present paper, from Eqs. 447) and (63), we obtain the explicit expression for $\hat{\Xi}$ as

$$
\hat{\Xi}=e^{i \varphi} \sqrt{\frac{n_{0}}{n V}} \hat{b}_{0}=e^{i \varphi} \sqrt{\frac{n_{0}}{n V}} e^{i \hat{G}(\mathbf{y})} \hat{a}_{0} e^{-i \hat{G}(\mathbf{y})}
$$

From Eqs. (49) and (62), we confirm that

$$
\hat{\Xi}|N, \mathbf{y}\rangle=\Xi|N-1, \mathbf{y}\rangle,
$$

which was assumed in Ref. [15]. The operator $\hat{\Xi}$ characterizes the condensation by having a finite matrix element [15]. In the following we will reveal a striking property of $\hat{\Xi}$ (or, equivalently, $\hat{b}_{0}$ ); it is a "natural coordinate" of interacting bosons.

On the other hand, other operators, which also characterize the condensation, were introduced in Refs. 10,17, 18]. Girardeau and Arnowitt [10] defined $\hat{\beta}_{0} \equiv \hat{a}_{0} \hat{N}_{0}^{-1 / 2}$, Gardiner [17] introduced $\hat{A}=\hat{a}_{0}\left(\hat{N}_{0} / \hat{N}\right)^{1 / 2}$ (which is an operator form of Eqs. (9) and (10) of Ref. [17]), and Castin and Dum 18 introduced $\hat{a}_{\Phi_{e x}} \equiv \int d r \Phi_{e x}^{*}(r, t) \hat{\psi}(r, t)$. These operators are totally different from $\hat{\Xi}$ or $\hat{b}_{0}$ because complicated many-particle correlations, which are included in $\hat{\Xi}$

and $\hat{b}_{0}$, are not included in $\hat{\beta}_{0}, \hat{A}$ and $\hat{a}_{\Phi_{e x}}$. For example, $\hat{a}_{\Phi_{e x}}$ is a linear combination of annihilation operators of free bosons, whereas $\hat{b}_{0}$ is a nonlinear function of both the annihilation and creation operators of free bosons. As a result, in contrast to Eqs. (49) and (67), application of either $\hat{\beta}_{0}, \hat{A}$ or $\hat{a}_{\Phi_{e x}}$ to the ground state of $N$ bosons does not yield the ground state of $N-1$ bosons; it yields an excited state which is not an eigenstate. Moreover, they are not a natural coordinate of interacting bosons in the sense explained in the following. Therefore, we do not use these operators, although they would be useful in other problems.

\section{Low-lying excited states}

Excited states of a fixed number of interacting bosons were discussed in Refs. 10,17, 18. In the present formulation, we may obtain low-lying excited states by the application to $|N, \mathbf{y}\rangle$ of functions of $\hat{b}_{\mathbf{k}}^{\top}$ 's with $\mathbf{k} \neq \mathbf{0}$. However, since we do not need any explicit expressions of the excited states in the following analysis, we do not seek for them in the present paper.

\section{TIME EVOLUTION OF BOSONS IN A LEAKY BOX}

The time evolution of a condensate(s) in an open box(es) was discussed previously for the cases of non-interacting bosons in Refs. [11 13 and for the case of two-mode interacting bosons in Ref. [23]. In the present paper, using $\hat{b}_{0}$, we study the case of infinite-mode interacting bosons.

\section{A. Gedanken experiment}

In most real systems, there is a finite probability of exchanging bosons between the box and the environment. Hence, even if one fixes $N$ at some time, $N$ will fluctuate at later times. Namely, the boson state undergoes a nonequilibrium time evolution when its number fluctuation is initially suppressed. To simulate this situation, we consider the following gedanken experiment [Fig. 1]. 


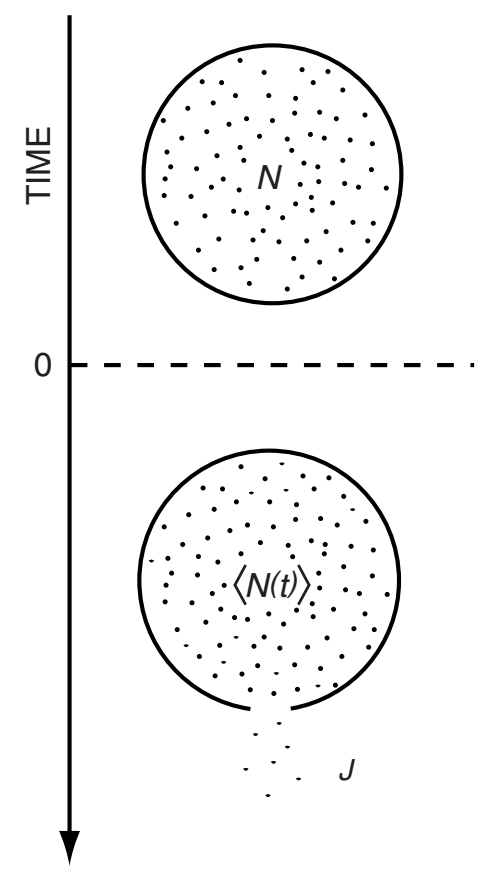

FIG. 1. Our gedanken experiment. $N$ bosons are confined in a closed box for $t<0$. At $t=0$ a small hole is made in the box, so that a small leakage flux $J$ is induced, and the expectation value $\langle N(t)\rangle$ of the number of bosons in the box decreases with time.

Suppose that bosons are confined in a box which is kept at zero temperature, and that the wall of the box is not permeable to the flow of the bosons, i.e., the probability of boson's permeating through the wall within a time scale of our interest is negligible. If one measures the number of the bosons at a time $t=t_{p}(<0)$, and if the box is kept closed until $t=0$, then the density operator $\hat{\rho}(t)$ of the bosons in the box for $t_{p}<t<0$ is

$$
\hat{\rho}(t)=|N, \mathbf{y}\rangle\langle N, \mathbf{y}| \text { for } t_{p}<t<0 .
$$

Assume that this box is placed in a large room, which has no bosons initially. Suppose now that at $t=0$ he makes a small hole(s), or slightly lowers the potential of the wall of the box, so that a small but finite flow $J$ of the bosons from the inside to the outside of the box becomes possible for $t \geq 0$. We study the time evolution for $t \geq 0$ of the density operator $\hat{\rho}(t)$ of the bosons in the box.

The expectation value of $N$ will be a decreasing function of $t$, which we denote $\langle N(t)\rangle($ hence $\langle N(0)\rangle=N)$. It is obvious that as $t \rightarrow \infty$ the system approaches the equilibrium state. Therefore, we are most interested in the early stage of the time evolution, for which

$$
|N-\langle N(t)\rangle| \ll N
$$

Note that if $J$ were not small enough, then the state in the box would evolve into a nonequilibrium excited state. The property of such a nonequilibrium state would depend strongly on details of the structures of the box and the hole or wall. In the present paper, we are not interested in such structure-sensitive states. Therefore, we assume that $J$ is small enough that only transitions between the ground states for different values of $N$ are possible.

\section{B. Total Hamiltonian}

Let $\mathcal{V}$ denote the volume of the room, which is much larger than the volume $V$ of the box;

$$
\mathcal{V} \gg V \text {. }
$$

The total boson field $\hat{\psi}^{\text {total }}(\mathbf{r})$ is defined on $\mathcal{V}$,

$$
\hat{\psi}^{\text {total }}(\mathbf{r})=\hat{\psi}(\mathbf{r})+\hat{\psi}^{E}(\mathbf{r})
$$


where $\hat{\psi}(\mathbf{r})$ is localized in the box, and $\hat{\psi}^{E} \equiv \hat{\psi}^{\text {total }}-\hat{\psi}$ is the boson field of "environment." Then, the total Hamiltonian may take the following form:

$$
\hat{H}^{\text {total }}=\hat{H}+\hat{H}^{E}+\hat{H}^{E S},
$$

where $\hat{H}$ and $\hat{H}^{E}$ are the Hamiltonians of the $\hat{\psi}$ and $\hat{\psi}^{E}$ fields, respectively, when they are closed. Small but finite amplitudes of scattering between the $\hat{\psi}$ and $\hat{\psi}^{E}$ fields are caused by the residual Hamiltonian $\hat{H}^{E S}$. Under our assumption (8), the probability of multi-particle collisions during the escape from the box is negligible. Therefore, $\hat{H}^{E S}$ should take the following form:

$$
\hat{H}^{E S}=\lambda \int d^{3} r \hat{\psi}^{E \dagger}(\mathbf{r}) f(\mathbf{r}) \hat{\psi}(\mathbf{r})+\text { h.c. },
$$

where $\lambda$ is a constant which has the dimension of energy, and $f(\mathbf{r})$ is a dimension-less function which takes values of order unity when $\mathbf{r}$ is located in the boundary region between the box and the environment, and $f(\mathbf{r})=0$ otherwise. Although the value of $\lambda$ and the form of $f$ depend on the structures of the box and the hole or walls, our final results (e.g., Eq. (107)) are independent of such details.

\section{Low-lying states of the total system}

We here list states of the total system which are relevant to the following analysis. Since $\hat{H}^{E S}$ is weak, quasi eigenstates of the total system are well approximated by the products of eigenstates of the box and of the environment. Recall that $|N-\langle N(t)\rangle| \ll N$ for the time interval of our interest, and that $J$ is small enough so that only transitions between the ground states for different values of $N$ are possible. Therefore, among many possible states of the box the relevant states are $|N-\Delta N, \mathbf{y}\rangle$ 's with $|\Delta N| \ll N$. On the other hand, there are no bosons in the environment at $t<0$. That is, the environment is initially in the vacuum, which we denote $\left|0^{E}\right\rangle$. Hence, from Eq. (68), the initial density operator of the total system is

$$
\hat{\rho}^{\text {total }}(t)=|N, \mathbf{y}\rangle\left|0^{E}\right\rangle\left\langle 0^{E}\right|\langle N, \mathbf{y}| \quad(t<0) .
$$

Bosons escape from the box into the environment for $t \geq 0$. Since $\mathcal{V} \gg V$, the boson density of the environment is kept essentially zero, and BEC does not occur in the environment, for the time period of Eq. (69). We can therefore take the simple number states $\left|n_{\mathbf{k}}^{E}, n_{\mathbf{k}^{\prime}}^{E}, \cdots\right\rangle$ 's of free bosons as eigenstates of the environment, where $n_{\mathbf{k}}$ denotes the number of bosons in mode $\mathbf{k}$. For example, we shall write $\left|1_{\mathbf{k}}^{E}\right\rangle$ to denote the environment state in which mode $\mathbf{k}$ are occupied by a single boson whereas the other modes are empty. Therefore, the relevant states of the total system, i.e., low-lying quasi eigenstates of $\hat{H}^{\text {total }}$, can be written as

$$
|N-\Delta N, \mathbf{y}\rangle\left|n_{\mathbf{k}}^{E}, n_{\mathbf{k}^{\prime}}^{E}, \cdots\right\rangle \quad(|\Delta N| l N),
$$

where, since $\hat{H}^{\text {total }}$ conserves the total number of bosons,

$$
\Delta N=\sum_{\mathbf{k}} n_{\mathbf{k}}^{E}
$$

\section{Time evolution in a short time interval $\Delta t$}

We are interested in the reduced density operator of bosons in the box for $t \geq 0$;

$$
\hat{\rho}(t) \equiv \operatorname{Tr}^{\mathrm{E}}\left[\hat{\rho}^{\text {total }}(t)\right],
$$

where $\operatorname{Tr}^{\mathrm{E}}$ is the trace operation over the environment degrees of freedom. The expectation value of any observable $\hat{Q}$ in the box can be evaluated from $\hat{\rho}(t)$ as

$$
\begin{aligned}
\langle Q(t)\rangle & \equiv \operatorname{Tr}^{\text {total }}\left[\hat{\rho}^{\text {total }}(t) \hat{Q}\right] \\
& =\operatorname{Tr}[\hat{\rho}(t) \hat{Q}]
\end{aligned}
$$


where $\operatorname{Tr}$ denotes the trace operation over the degrees of freedom in the box. Equation (74) yields

$$
\begin{aligned}
\hat{\rho}(0) & =|N, \mathbf{y}\rangle\langle N, \mathbf{y}| \\
\langle Q(0)\rangle & =\langle N, \mathbf{y}|\hat{Q}| N, \mathbf{y}\rangle .
\end{aligned}
$$

Although we may evaluate $\hat{\rho}(t)$ by solving a master equation, we here present a different (but equivalent) method, by which the physical meaning can be seen clearly. We begin with noting that a single action of $\hat{H}^{E S}$ of Eq. (73) can only change $\left(N, N^{E}\right)$ by either $(-1,+1)$ or $(+1,-1)$, and that the latter change is impossible for the initial density operator (74). Therefore, after a short time interval $\Delta t$ which satisfies $J \Delta t \ll 1$, the state vector $|N, \mathbf{y}\rangle\left|0^{E}\right\rangle$ evolves into a state of the following form:

$$
e^{-i E_{N, \mathbf{y}} \Delta t / \hbar}|N, \mathbf{y}\rangle\left|0^{E}\right\rangle+\sum_{\mathbf{k}} c_{\mathbf{k}}^{(1)}(\Delta t) e^{-i\left(E_{N-1, \mathbf{y}}+\epsilon_{k}^{(0)}\right) \Delta t / \hbar}|N-1, \mathbf{y}\rangle\left|1_{\mathbf{k}}^{E}\right\rangle+O\left(\lambda^{2}\right),
$$

where

$$
\begin{aligned}
c_{\mathbf{k}}^{(1)}(\Delta t) & \equiv \frac{1}{i \hbar} \int_{0}^{\Delta t} M_{\mathbf{k}} e^{i\left(\epsilon_{k}^{(0)}-\mu\right) \tau / \hbar} d \tau, \\
M_{\mathbf{k}} & \equiv\left\langle 1_{\mathbf{k}}^{E}\left|\left\langle N-1, \mathbf{y}\left|\hat{H}^{E S}\right| N, \mathbf{y}\right\rangle\right| 0^{E}\right\rangle, \\
\mu & \equiv E_{N, \mathbf{y}}-E_{N-1, \mathbf{y}}
\end{aligned}
$$

Therefore, the reduced density operator is evaluated as

$$
\hat{\rho}(\Delta t)=w(0 ; \Delta t)|N, \mathbf{y}\rangle\langle N, \mathbf{y}|+w(1 ; \Delta t)| N-1, \mathbf{y}\rangle\langle N-1, \mathbf{y}|+O\left(\lambda^{3}\right),
$$

where

$$
\begin{aligned}
& w(0 ; \Delta t) \equiv 1-w(1 ; \Delta t), \\
& w(1 ; \Delta t) \equiv \sum_{\mathbf{k}}\left|c_{\mathbf{k}}^{(1)}(\Delta t)\right|^{2} .
\end{aligned}
$$

Here, we have normalized $\hat{\rho}(\Delta t)$ to order $\lambda^{2}$ by Eq. (86). We now take $\Delta t$ in such a way that

$$
\hbar / E_{c}<\Delta t \ll 1 / J
$$

where $E_{c}$ is the energy range of $\epsilon_{\mathbf{k}}^{(0)}$ in which $\left|M_{\mathbf{k}}\right|^{2}$ is finite and approximately constant. Then, since $\mathbf{k}$ of the environment takes quasi-continuous values, $w(1 ; \Delta t)$ becomes proportional to $\Delta t$ :

$$
w(1 ; \Delta t)=J \Delta t
$$

To evaluate $J$, we calculate $\left|M_{\mathbf{k}}\right|^{2}$ using Eqs. (56) and (73) as

$$
\begin{aligned}
\left|M_{\mathbf{k}}\right|^{2} & =\left|\lambda \int d^{3} r f(\mathbf{r})\left\langle 1_{\mathbf{k}}^{E}\left|\left\langle N-1, \mathbf{y}\left|\hat{\psi}^{E \dagger}(\mathbf{r}) \hat{\Xi}\right| N, \mathbf{y}\right\rangle\right| 0^{E}\right\rangle\right|^{2} \\
& =N \frac{n_{0}}{n}\left|\frac{\lambda}{\sqrt{V}} \int d^{3} r f(\mathbf{r}) \varphi_{\mathbf{k}}^{E *}(\mathbf{r})\right|^{2}
\end{aligned}
$$

where $\varphi_{\mathbf{k}}^{E}(\mathbf{r})$ is the mode function of mode $\mathbf{k}$ of the environment. Regarding the volume dependence, $\varphi_{\mathbf{k}}^{E}$ behaves as $\sim 1 / \sqrt{\mathcal{V}}$, whereas $f$ is localized in the boundary region, whose volume is denoted by $v$, of the box and the environment. Therefore,

$$
\left|M_{\mathbf{k}}\right|^{2} \approx \frac{n_{0}}{n}|\lambda|^{2} \frac{v^{2}}{V \mathcal{V}}
$$

On the other hand, from Eq. (35), an escaping boson has the energy of $g n$. The density of states of the environment at this energy is

$$
\frac{\mathcal{V}}{2 \pi^{2} \hbar^{3}} \sqrt{\frac{m^{3} g n}{2}}
$$


Therefore, the leakage flux $J$ is estimated as

$$
J \approx N \frac{n_{0}}{n} \frac{v}{V} \frac{|\lambda|^{2} v}{\hbar^{4}} \sqrt{m^{3} g n}=\frac{n_{0}|\lambda|^{2} v^{2}}{\hbar^{4}} \sqrt{m^{3} g n},
$$

where numerical factors of order unity have been absorbed in $\lambda$. We observe that $J$ is reduced by the factor $v / V$ $(\ll 1)$, which means that the escape process is a "surface effect", i.e., it occurs only in the boundary region. On the other hand, $J$ is enhanced by the factor $N(\gg 1)$. This enhancement is typical to the boson condensation.

From our assumption of small $J$, the rhs of Eq. (93) should be smaller than the critical value $J_{c r}$ of the flux above which bosons in the box get excited [24]. It is seen that this condition is satisfied when $v$ and/or $|\lambda|$ is small enough.

\section{E. Time evolution for $0 \leq J t \ll N$}

We have found in the previous subsection that the reduced density operator $\hat{\rho}$ evolves from the pure state $(79)$ to the mixed state 85$)$ after a small time interval $\Delta t(\ll 1 / J)$. Since the latter is a classical mixture of two different states, $|N, \mathbf{y}\rangle\langle N, \mathbf{y}|$ and $|N-1, \mathbf{y}\rangle\langle N-1, \mathbf{y}|$, we can separately solve the time evolution for each state. For each state, further transitions occur in the subsequent time intervals, $(\Delta t, 2 \Delta t],(2 \Delta t, 3 \Delta t], \cdots$. Since $\mathcal{V} \gg V$, the recursion time of an escaped boson to return to the original position is extremely long (except for rare events whose probability $\rightarrow 0$ as $\mathcal{V} \rightarrow \infty$.) Therefore, as long as $J \ll J_{c r}$, we can neglect any quantum as well as classical correlations between transitions of different time intervals. This allows us to take the no-boson state, $\left|0^{E}\right\rangle$, as the initial state of the environment for each time interval $(\ell \Delta t,(\ell+1) \Delta t]$, where $\ell=0,1,2, \cdots$. Hence, for every time interval, we may use the same formula (85). Furthermore, we can neglect the $N$ dependencies of $w$ and $J$ under our assumption of Eq. (69). Therefore,

$$
\begin{aligned}
& \hat{\rho}(2 \Delta t) \\
& =w(0 ; \Delta t)\{w(0 ; \Delta t)|N, \mathbf{y}\rangle\langle N, \mathbf{y}|+w(1 ; \Delta t)| N-1, \mathbf{y}\rangle\langle N-1, \mathbf{y}|\} \\
& \quad+w(1 ; \Delta t)\{w(0 ; \Delta t)|N-1, \mathbf{y}\rangle\langle N-1, \mathbf{y}|+w(1 ; \Delta t)| N-2, \mathbf{y}\rangle\langle N-2, \mathbf{y}|\} \\
& =w(0 ; 2 \Delta t)|N, \mathbf{y}\rangle\langle N, \mathbf{y}| \\
& \quad+w(1 ; 2 \Delta t)|N-1, \mathbf{y}\rangle\langle N-1, \mathbf{y}| \\
& \quad+w(2 ; 2 \Delta t)|N-2, \mathbf{y}\rangle\langle N-2, \mathbf{y}|,
\end{aligned}
$$

where

$$
\begin{aligned}
w(0 ; 2 \Delta t) & \equiv w(0 ; \Delta t)^{2} \\
& =(1-J \Delta t)^{2} \\
w(1 ; 2 \Delta t) & \equiv w(0 ; \Delta t) w(1 ; \Delta t)+w(1 ; \Delta t) w(0 ; \Delta t) \\
& =2(1-J \Delta t) J \Delta t \\
w(2 ; 2 \Delta t) & \equiv w(1 ; \Delta t) w(1 ; \Delta t) \\
& =J^{2}(\Delta t)^{2} .
\end{aligned}
$$

The time evolution in the subsequent times can be calculated in a similar manner. Let

$$
t=M \Delta t
$$

where $M(<N)$ is a positive integer. We find

$$
\hat{\rho}(t)=\sum_{m=0}^{M} w(m ; t)|N-m, \mathbf{y}\rangle\langle N-m, \mathbf{y}|,
$$

where $w(m ; t)$ is the binomial distribution;

$$
w(m ; t)=\left(\begin{array}{c}
M \\
m
\end{array}\right)(1-J \Delta t)^{M-m}(J \Delta t)^{m} .
$$

We find from Eq. (99) that $w(m ; t)$ is the probability of finding $N-m$ bosons in the box at $t$. From the conservation of the total number of bosons, Eq. (76), this probability equals the probability that $m$ bosons have escaped from the box by the time $t$. Using Eq. (100), we find 


$$
\begin{aligned}
\langle N(t)\rangle & =\operatorname{Tr}[\hat{\rho}(t) \hat{N}]=\sum_{m=0}^{M} w(m ; t)(N-m)=N-J t \\
\left\langle N^{E}(t)\right\rangle & =\operatorname{Tr}\left[\hat{\rho}(t) \hat{N}^{E}\right]=\sum_{m=0}^{M} w(m ; t) m=J .
\end{aligned}
$$

Since $E_{c}$ in Eq. (88) is of the order of the atomic energy, we can take $\Delta t$ extremely small such that

$$
M \gg 1 \text { and } M \gg J t
$$

for a finite $t$ that satisfies Eq. (69). In this case, Eq. (100) can be approximated by the Poisson distribution;

$$
w(m ; t) \approx K(M, J t) e^{-J t} \frac{(J t)^{m}}{m !}
$$

Here, $K$ is the normalization factor,

$$
\frac{1}{K(M, x)} \equiv e^{-x} \sum_{m=0}^{M} \frac{x^{m}}{m !},
$$

which approaches unity for all $x$ as $M \rightarrow \infty$. For large but finite $M$, we can easily show that

$$
\left|\frac{1}{K(M, x)}-1\right| \sim \frac{e^{-x}}{\sqrt{2 \pi(M+1)}}\left(\frac{e x}{M+1}\right)^{M+1} .
$$

Therefore, under the condition (103), we can take $K(M, J t)=1$ to a very good approximation, and we henceforth drop $K$ from Eq. (104). Furthermore, since $w(m, t) \approx 0$ for $m \gg J t$, we may extend the summation of Eq. (99) to $N$. We thus obtain

$$
\begin{aligned}
\hat{\rho}(t) & \approx e^{-J t} \sum_{m=0}^{N} \frac{(J t)^{m}}{m !}|N-m, \mathbf{y}\rangle\langle N-m, \mathbf{y}| \\
& =e^{-J t} \sum_{m=0}^{N} \frac{(J t)^{N-m}}{(N-m) !}|m, \mathbf{y}\rangle\langle m, \mathbf{y}| .
\end{aligned}
$$

Since this final result is valid even at $t=0$ (despite our use of the assumption $M \gg 1$ ), it is valid for all $t$ as long as

$$
0 \leq J t \ll N \text { and } N \gg 1
$$

Note that the final result (107) is quite general because all the details of the box-environment interaction $\hat{H}^{E S}$ have been absorbed in $J$.

The probability $P(m, t)$ of finding $m$ bosons in the box at $t$ is evaluated as

$$
\begin{aligned}
P(m, t) & =w(N-m ; t) \\
& =e^{-J t} \frac{(J t)^{N-m}}{(N-m) !} .
\end{aligned}
$$

We call this distribution the "shifted Poisson distribution", because it is obtained by shifting the center of the Poisson distribution. The expectation values and variances are evaluated as

$$
\begin{aligned}
\langle N(t)\rangle & =N-J t \\
\left\langle N^{E}(t)\right\rangle & =J t \\
\left\langle\delta N(t)^{2}\right\rangle & =\left\langle\delta N^{E}(t)^{2}\right\rangle=J t .
\end{aligned}
$$




\section{NUMBER VERSUS PHASE}

\section{A. Cosine and sine operators of interacting many bosons}

Roughly speaking, the conjugate observable of the number is the phase. More precisely, however, physical observables are not the phase itself, but the cosine and sine of the phase. Namely, any physical measurement of a phase actually measures the cosine or sine of the phase 20]. In the case of a single-mode boson (i.e., a harmonic oscillator of a single degrees of freedom), it has been discussed that various definitions are possible for the cosine and sine operators [25]. This ambiguity does not matter in our case, because we are treating the case where the number of bosons is extremely large, whereas differences among different definitions appear only when the number of bosons is small. On the other hand, the crucial point in our case is how to select a single "coordinate" (dynamical variable) with which the phase is defined, among a huge degrees of freedom. To find such a "proper coordinate" is generally very difficult in many-body interacting systems.

Fortunately, we find that $\hat{b}_{0}$ is the proper coordinate of interacting bosons, with which we can successfully define the cosine and sine operators of interacting many bosons by

$$
\begin{aligned}
\cos \phi & \equiv \frac{1}{2 \sqrt{\hat{b}_{0}^{\dagger} \hat{b}_{0}+1}} \hat{b}_{0}+\hat{b}_{0}^{\dagger} \frac{1}{2 \sqrt{\hat{b}_{0}^{\dagger} \hat{b}_{0}+1}} \\
\sin \phi & \equiv \frac{1}{2 i \sqrt{\hat{b}_{0}^{\dagger} \hat{b}_{0}+1}} \hat{b}_{0}-\hat{b}_{0}^{\dagger} \frac{1}{2 i \sqrt{\hat{b}_{0}^{\dagger} \hat{b}_{0}+1}}
\end{aligned}
$$

These are the same forms as those of a single harmonic oscillator 25. In our case, however, there are a huge degrees of freedom with mutual interactions. As a result, the Hamiltonian does not take the simple bilinear form with respect to $\hat{b}_{0}$, hence the motion of $\hat{b}_{0}+\hat{b}_{0}^{\dagger}$ is not that of a harmonic-oscillator coordinate. Nevertheless, many formulas for the single mode case are applicable if they are based only on the commutation relations of a boson operator. In particular, owing to Eq. (50), we can treat any states that have the form of $\sum_{m} C_{m} \mid N-m$, y $\rangle$ as if we were treating a single-mode problem.

It will turn out in the following discussions that the above operators give reasonable results for the quantum phase of interacting bosons.

\section{B. Number and phase fluctuations of $|N, \mathbf{y}\rangle$}

As we have shown in section III, the ground state of a fixed number of bosons $|N, \mathbf{y}\rangle$ can be represented simply as a number state if we use a "natural coordinate" $\hat{b}_{0}$. Note that this state has a finite fluctuation of $\hat{a}_{0}^{\dagger} \hat{a}_{0}$ due to many-body interactions. Nevertheless, the total number of bosons $\hat{N}$ has a definite value;

$$
\begin{aligned}
\langle N\rangle_{N, \mathbf{y}} & \equiv\langle N, \mathbf{y}|\hat{N}| N, \mathbf{y}\rangle=N \\
\left\langle\delta N^{2}\right\rangle_{N, \mathbf{y}} & \equiv\left\langle N, \mathbf{y}\left|\delta \hat{N}^{2}\right| N, \mathbf{y}\right\rangle=0
\end{aligned}
$$

On the other hand, using the simple representation (48), we can easily show that

$$
\begin{aligned}
& \langle\cos \phi\rangle_{N, \mathbf{y}} \equiv\langle N, \mathbf{y}|\hat{\cos } \phi| N, \mathbf{y}\rangle=0 \\
& \langle\sin \phi\rangle_{N, \mathbf{y}} \equiv\langle N, \mathbf{y}|\sin \phi| N, \mathbf{y}\rangle=0
\end{aligned}
$$

Therefore, the ground state of a fixed number of bosons does not have a definite phase [20], as expected from the number-phase uncertainty relation, Eq. (38). It was sometimes argued that although $\hat{N}$ is definite the fluctuation of $\hat{a}_{0}^{\dagger} \hat{a}_{0}$ might allow for a definite phase [26]. However, our results (117) and (118) show explicitly that the fluctuation of $\hat{a}_{0}^{\dagger} \hat{a}_{0}$ does not help to develop a definite phase. Note that this is not due to our special choice of the cosine and sine operators, because the same conclusion is obtained also when the cosine and sine operators of $\hat{a}_{0}$ are used instead of Eqs. (113) and (114). We will touch on this point again in section VII. 


\section{Coherent state of interacting bosons}

We define a coherent state of interacting bosons (CSIB) by

$$
|\alpha, \mathbf{y}\rangle \equiv e^{-|\alpha|^{2} / 2} \sum_{m=0}^{\infty} \frac{\alpha^{n}}{\sqrt{m !}}|m, \mathbf{y}\rangle
$$

which is labeled by $\mathbf{y}$ and a complex number,

$$
\alpha \equiv e^{i \phi} \sqrt{N}
$$

The inverse transformation is

$$
|N, \mathbf{y}\rangle=\int_{-\pi}^{\pi} \frac{d \phi}{2 \pi}|\alpha, \mathbf{y}\rangle
$$

Regarding the number and phase fluctuations, we can easily show that $|\alpha, \mathbf{y}\rangle$ has the same properties as a coherent state of a single-mode harmonic oscillator [25]. Namely,

$$
\begin{gathered}
\langle N\rangle_{\alpha, \mathbf{y}} \equiv\langle\alpha, \mathbf{y}|\hat{N}| \alpha, \mathbf{y}\rangle=|\alpha|^{2}=N \\
\left\langle\delta N^{2}\right\rangle_{\alpha, \mathbf{y}} \equiv\left\langle\alpha, \mathbf{y}\left|\delta \hat{N}^{2}\right| \alpha, \mathbf{y}\right\rangle=|\alpha|^{2}=N
\end{gathered}
$$

and, for $|\alpha|^{2}=N \gg 1$,

$$
\begin{aligned}
\langle\sin \phi\rangle_{\alpha, \mathbf{y}} & \equiv\langle\alpha, \mathbf{y}|\sin \phi| \alpha, \mathbf{y}\rangle \\
& =\left[1-1 /\left(8|\alpha|^{2}\right)+\cdots\right] \sin \phi \\
\left\langle\delta \sin ^{2} \phi\right\rangle_{\alpha, \mathbf{y}} & \equiv\left\langle\alpha, \mathbf{y}\left|(\delta \sin \phi)^{2}\right| \alpha, \mathbf{y}\right\rangle \\
& =\left(1 / 4|\alpha|^{2}\right)\left(1-\sin ^{2} \phi\right)+\cdots,
\end{aligned}
$$

and similar results for the cosine operator. It is customary to express the results for the sine and cosine operators symbolically as

$$
\begin{aligned}
\langle\phi\rangle_{\alpha, \mathbf{y}} & \approx \phi, \\
\left\langle\delta \phi^{2}\right\rangle_{\alpha, \mathbf{y}} & \approx 1 /\left(4|\alpha|^{2}\right)=1 /(4 N) .
\end{aligned}
$$

Therefore, $|\alpha, \mathbf{y}\rangle$ is the minimum-uncertainty state in the sense that it has the minimum allowable value of the number-phase uncertainty product (NPUP) [Eq. (38)];

$$
\left\langle\delta N^{2}\right\rangle_{\alpha, \mathbf{y}}\left\langle\delta \phi^{2}\right\rangle_{\alpha, \mathbf{y}} \approx 1 / 4
$$

The magnitude of the number fluctuation is conveniently measured with the "Fano factor" $F$, which is defined by

$$
F \equiv\left\langle\delta N^{2}\right\rangle /\langle N\rangle
$$

For $|\alpha, \mathbf{y}\rangle$, we find

$$
F_{\alpha, \mathbf{y}} \equiv \frac{\left\langle\delta N^{2}\right\rangle_{\alpha, \mathbf{y}}}{\langle N\rangle_{\alpha, \mathbf{y}}}=1
$$

Therefore, using $\hat{b}_{0}$, we have successfully constructed a very special state of interacting bosons, $|\alpha, \mathbf{y}\rangle$, whose Fano factor is exactly unity, and which has the minimum allowable value of the NPUP. This should be contrasted with Bogoliubov's ground state $\left|\alpha_{0}, \mathbf{y}^{c l}\right\rangle^{c l}$, for which

$$
F^{c l} \equiv \frac{\left\langle\delta N^{2}\right\rangle^{c l}}{\langle N\rangle^{c l}}=1+\frac{\sum_{\mathbf{q} \neq \mathbf{0}}\left(\sinh \left|y_{q}^{c l}\right|\right)^{4}}{\left|\alpha_{0}\right|^{2}+\sum_{\mathbf{q} \neq \mathbf{0}}\left(\sinh \left|y_{q}^{c l}\right|\right)^{2}}>1
$$

and the NPUP is larger than $1 / 4$. The CSIB should not be confused with $\left|\alpha_{0}, \mathbf{y}^{c l}\right\rangle^{c l}$. 


\section{Number-phase squeezed state of interacting bosons}

We define a new state $|\xi, N, \mathbf{y}\rangle$ by

$$
\begin{aligned}
|\xi, N, \mathbf{y}\rangle & \equiv \sqrt{K\left(N,|\xi|^{2}\right)} e^{-|\xi|^{2} / 2} \sum_{n=0}^{N} \frac{\xi^{*(N-n)}}{\sqrt{(N-n) !}}|m, \mathbf{y}\rangle \\
& =\sqrt{K\left(N,|\xi|^{2}\right)} e^{-|\xi|^{2} / 2} \sum_{n=0}^{N} \frac{\xi^{*(N-n)}}{\sqrt{(N-n) ! n !}}\left(\hat{b}_{0}^{\dagger}\right)^{n}|0, \mathbf{y}\rangle,
\end{aligned}
$$

which is labeled by $\mathbf{y}$ and a complex number,

$$
\xi \equiv e^{i \phi}|\xi|
$$

We henceforth assume that

$$
|\xi|^{2} \ll N \text { and } N \gg 1,
$$

which allows us to set $K\left(N,|\xi|^{2}\right)=1$ to a very good approximation.

The probability $P(m)$ of finding $m$ bosons for the state $|\xi, N, \mathbf{y}\rangle$ obeys the shifted Poisson distribution [cf. Eq. (109)],

$$
P(m)=e^{-|\xi|^{2}} \frac{|\xi|^{2(N-m)}}{(N-m) !} .
$$

The number fluctuation and the Fano factor are evaluated as

$$
\begin{aligned}
\langle N\rangle_{\xi, N, \mathbf{y}} & \equiv\langle\xi, N, \mathbf{y}|\hat{N}| \xi, N, \mathbf{y}\rangle=N-|\xi|^{2}, \\
\left\langle\delta N^{2}\right\rangle_{\xi, N, \mathbf{y}} & \equiv\left\langle\xi, N, \mathbf{y}\left|\delta \hat{N}^{2}\right| \xi, N, \mathbf{y}\right\rangle=|\xi|^{2}, \\
F_{\xi, N, \mathbf{y}} & \equiv \frac{\left\langle\delta N^{2}\right\rangle_{\xi, N, \mathbf{y}}}{\langle N\rangle_{\xi, N, \mathbf{y}}}=\frac{|\xi|^{2}}{N-|\xi|^{2}} \approx \frac{|\xi|^{2}}{N} \ll 1 .
\end{aligned}
$$

As compared with Eqs. (123) and (130), we observe that the state $|\xi, N, \mathbf{y}\rangle$ has a very narrow distribution of the boson number. On the other hand, $|\xi, N, \mathbf{y}\rangle$ has a well-defined phase [20] when

$$
1 \ll|\xi|^{2} \ll N
$$

In fact, under this condition we can easily show that

$$
\begin{aligned}
\langle\sin \phi\rangle_{\xi, N, \mathbf{y}} & \equiv\langle\xi, N, \mathbf{y}|\sin \phi| \xi, N, \mathbf{y}\rangle \\
& =\left[1-1 /\left(8|\xi|^{2}\right)+\cdots\right] \sin \phi \\
\left\langle\delta \sin ^{2} \phi\right\rangle_{\xi, N, \mathbf{y}} & \equiv\left\langle\xi, N, \mathbf{y}\left|(\delta \sin \phi)^{2}\right| \xi, N, \mathbf{y}\right\rangle \\
& =\left(1 / 4|\xi|^{2}\right)\left(1-\sin ^{2} \phi\right)+\cdots,
\end{aligned}
$$

and similar results for the cosine operator. As in the case of the CSIB, we may express these results symbolically as

$$
\begin{aligned}
\langle\phi\rangle_{\xi, N, \mathbf{y}} & \approx \phi \\
\left\langle\delta \phi^{2}\right\rangle_{\xi, N, \mathbf{y}} & \approx 1 /\left(4|\xi|^{2}\right) .
\end{aligned}
$$

Therefore, just as $|\alpha, \mathbf{y}\rangle$ does, $|\xi, N, \mathbf{y}\rangle$ has the minimum value of the NPUP;

$$
\left\langle\delta N^{2}\right\rangle_{\xi, N, \mathbf{y}}\left\langle\delta \phi^{2}\right\rangle_{\xi, N, \mathbf{y}} \approx 1 / 4 \quad\left(\text { for } 1 \ll|\xi|^{2} \ll N\right) .
$$

Since each component of the product satisfies $\left\langle\delta \hat{N}^{2}\right\rangle_{\xi, N, \mathbf{y}} \ll\left\langle\delta \hat{N}^{2}\right\rangle_{\alpha, \mathbf{y}}$ and $\left\langle\delta \phi^{2}\right\rangle_{\xi, N, \mathbf{y}} \gg\left\langle\delta \phi^{2}\right\rangle_{\alpha, \mathbf{y}},|\xi, N, \mathbf{y}\rangle$ is obtained by "squeezing" $|\alpha, \mathbf{y}\rangle$ in the direction of $\hat{N}$, while keeping the NPUP minimum. ( $c f$. The conventional squeezed state has a larger NPUP [25].) We thus call $|\xi, N, \mathbf{y}\rangle$ the "number-phase squeezed state of interacting bosons" (NPIB). 


\section{E. Phase-randomized mixture of number-phase squeezed states of interacting bosons}

We now take

$$
\xi=e^{i \phi} \sqrt{J t} \equiv \xi(t)
$$

That is, $|\xi|^{2}=J t$. Then, inequalities (135) are satisfied because of our assumption (108). We can show by explicit calculation that Eq. (107) can be rewritten as

$$
\hat{\rho}(t)=\int_{-\pi}^{\pi} \frac{d \phi}{2 \pi}\left|e^{i \phi} \sqrt{J t}, N, \mathbf{y}\right\rangle\left\langle e^{i \phi} \sqrt{J t}, N, \mathbf{y}\right| .
$$

Therefore, the boson state in the box can be viewed either as the shifted Poissonian mixture, Eq. (107), of NSIBs, or as the phase-randomized mixture (PRM), Eq. (147), of NPIBs. Both representations are simply described in terms of $\hat{b}_{0}$. In contrast, the same $\hat{\rho}(t)$ would be described in an very complicated manner in terms of bare operators.

We have thus obtained double pictures (or representations), Eqs. (107) and (147), for the same physical state 27,28]. According to the former picture, the state of the box is one of NSIBs, for which the number of bosons is definite (but unknown), whereas the phase is completely indefinite. According to the latter picture, on the other hand, the state is one of NPIBs, for which the number of bosons has a finite fluctuation $\left\langle\delta N^{2}\right\rangle \approx J t$, whereas the phase is almost definite [20] (but unknown), $\left\langle\delta \phi^{2}\right\rangle \approx 1 /(4 J t)$. What allows these double pictures is the superposition principle 28].

In addition to Eqs. (107) and (147), there are many other ways to express $\hat{\rho}(t)$ as different mixtures. Among them, Eq. (107) is the form in which each element of the mixture has the smallest value of the number fluctuation, whereas in Eq. (147) each element of the mixture has the smallest value of the phase fluctuation. Therefore, the latter representation is particularly convenient for discussing physical properties that are related to the phase, as will be shown in sections VIB and VII.

\section{F. Origin of the direction of the time evolution}

As the time evolves, the number of bosons decreases as

$$
\langle N(t)\rangle=N-J t
$$

As a result, the energy of the bosons in the box decreases with $t$. For example, for each element of Eq. (147),

$$
\left\langle e^{i \phi} \sqrt{J t}, N, \mathbf{y}|\hat{H}| e^{i \phi} \sqrt{J t}, N, \mathbf{y}\right\rangle<\langle N, \mathbf{y}|\hat{H}| N, \mathbf{y}\rangle \quad \text { for } t>0 .
$$

However, we note that this energy difference is just a consequence of the difference in $\langle N\rangle$. Namely, $|N, \mathbf{y}\rangle$ and the NPIB have the same energy if they have the same value of $\langle N\rangle$;

$$
\left\langle e^{i \phi} \sqrt{J t}, N+J t, \mathbf{y}|\hat{H}| e^{i \phi} \sqrt{J t}, N+J t, \mathbf{y}\right\rangle \approx\langle N, \mathbf{y}|\hat{H}| N, \mathbf{y}\rangle
$$

We can therefore conclude that the direction of the time evolution, from $|N, \mathbf{y}\rangle$ to the $\mathrm{PRM}$ of $|\xi, N, \mathbf{y}\rangle$, is not determined by an energy difference. Hence, it must be due to difference in the nature of the wavefunctions. The study of such a nature, however, needs many pages of analysis, which is beyond the scope of this paper, thus will be described elsewhere 29].

\section{ACTION OF MEASUREMENT OR ITS EQUIVALENCE}

In the previous section, we have obtained the double pictures, Eqs. (107) and (147). Depending on the physical situation, either picture is convenient [28]. To explain this point, we discuss two examples in this section. 


\section{A. Number measurement}

Suppose that one measures $N$ of the boson system whose density operator is given by Eq. (107), or, equivalently, by Eq. (147). In this case, the former expression is convenient. In fact, if the measurement error

$$
\delta N_{\text {err }}<\sqrt{\left\langle\delta N(t)^{2}\right\rangle}=\sqrt{J t},
$$

and if the measurement is of the first kind [30], then the action of the measurement is to narrower the number distribution as small as $\delta N_{\text {err }}$, of the rhs of Eq. (107). That is, the density operator immediately after the measurement is generally given by [31]

$$
\hat{\rho}_{\bar{N}}(t) \equiv \sum_{m=0}^{N} W(m-\bar{N})|m, \mathbf{y}\rangle\langle m, \mathbf{y}|
$$

Here, $\bar{N}$ is the value of $\phi$ obtained by the measurement, and $W$ is a smooth function which has the following properties:

$$
\begin{aligned}
& W(m-\bar{N}) \geq 0, \\
& W(m-\bar{N}) \approx 0 \text { for }|m-\bar{N}| \gtrsim \delta N_{e r r}, \\
& \sum_{m=0}^{N} W(m-\bar{N})=1 .
\end{aligned}
$$

The detailed form of $W$ depends on the detailed structures of the measuring apparatus, and thus is of no interest here. In an ideal case where $\delta N_{e r r} \rightarrow 0, W$ becomes Kronecker's delta, and

$$
\hat{\rho}_{\bar{N}}(t) \rightarrow|\bar{N}, \mathbf{y}\rangle\langle\bar{N}, \mathbf{y}| \cdot
$$

On the other hand, if the measurement is rather inaccurate in such a way that

$$
\delta N_{e r r}>\sqrt{\left\langle\delta N(t)^{2}\right\rangle}=\sqrt{J t},
$$

then almost no change of $\hat{\rho}$ is induced by the measurement, if it is of the first kind [31].

\section{B. Phase measurement}

Suppose that one measures $\phi$ of the boson system whose density operator is Eq. (107), or, equivalently, Eq. (147). In this case, the latter representation is convenient. In fact, if the measurement error

$$
\delta \phi_{e r r}>\sqrt{\left\langle\delta \phi^{2}\right\rangle_{\xi, N, \mathbf{y}}}=\frac{1}{2|\xi(t)|}=\frac{1}{2 \sqrt{J t}}
$$

and if the measurement is performed in such a way that the backaction of the measurement is minimum, then the action of the measurement is just to find (or, get to know) the "true" value of $\phi$, to the accuracy of $\delta \phi_{e r r}$, among many possibilities in the rhs side of Eq. (147). Therefore, the density operator just after the measurement is generally given by [31]

$$
\hat{\rho}_{\bar{\phi}}(t) \equiv \int_{-\pi}^{\pi} \frac{d \phi}{2 \pi} D(\phi-\bar{\phi})\left|e^{i \phi} \sqrt{J t}, N, \mathbf{y}\right\rangle\left\langle e^{i \phi} \sqrt{J t}, N, \mathbf{y}\right|
$$

Here, $\bar{\phi}$ is the value of $\phi$ obtained by the measurement, and $D(\phi-\bar{\phi})$ is a smooth function which has the following properties:

$$
\begin{aligned}
& D(\phi-\bar{\phi}) \geq 0, \\
& D(\phi-\bar{\phi}) \approx 0 \text { for }|\phi-\bar{\phi}| \gtrsim \delta \phi_{e r r}, \\
& \int_{-\pi}^{\pi} \frac{d \phi}{2 \pi} D(\phi-\bar{\phi})=1 .
\end{aligned}
$$


The detailed form of $D$ depends on the detailed structures of the measuring apparatus, and thus is of no interest here.

On the other hand, if the measurement is very accurate in such a way that

$$
\delta \phi_{e r r}<\sqrt{\left\langle\delta \phi^{2}\right\rangle_{\xi, N, \mathbf{y}}}=\frac{1}{2|\xi(t)|}=\frac{1}{2 \sqrt{J t}}
$$

then $\hat{\rho}$ will "collapse" into another state whose phase fluctuation is less than $\left\langle\delta \phi^{2}\right\rangle_{\xi, N, \mathbf{y}}$. However, such an accurate measurement is practically difficult when $J t \gg 1$. Therefore, in most experiments we may take Eq. (159) for the density operator after the measurement, if the measurement is performed in such a way that the backaction of the measurement is minimum.

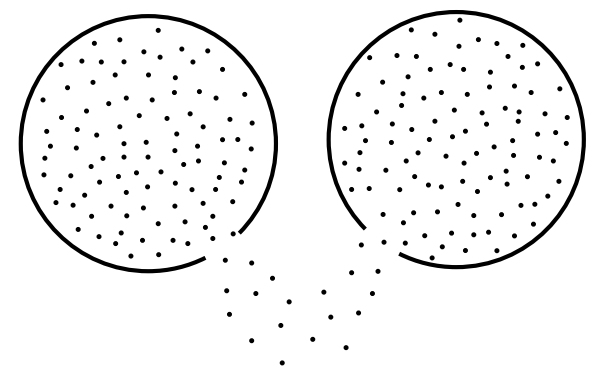

FIG. 2. Bosons are confined independently in two boxes. The number of bosons in each box is fixed for $t<0$. If holes are made in the boxes at $t>0$, then the leakage fluxes are induced, which exhibit interference.

For example, suppose that one prepares bosons independently in two boxes [Fig. 2], where the number of bosons in each box is fixed for $t<0$. At $t=0$ a small hole is made in each box, and small fluxes of bosons escape from the boxes. Since $\hat{\rho}$ of each box evolves as Eq. (147), it is clear that these fluxes can interfere at each experimental run (as in the cases of non-interacting bosons [11]13] and two-mode bosons [23]) as the interference between two NPIBs of two boxes. From the interference pattern, one can measure the relative phase $\bar{\phi}$ of two condensates. After the measurement, $\hat{\rho}$ of each box would take the form of eq. (159). Such an experiment would be possible by modifying the experiment of Ref. [32] in such a way that $J$ becomes small enough.

\section{ORDER PARAMETER}

The order parameter of BEC can be defined in various ways. For the ground state $\left|\alpha, \mathbf{y}^{c l}\right\rangle^{c l}$ of the semiclassical Hamiltonian $\hat{H}^{c l}$, different definitions give the same result. However, this is not the case for $|N, \mathbf{y}\rangle$ and $|\xi, N, \mathbf{y}\rangle$. We explore these points in this section.

\section{A. Off-diagonal long-range order}

We first consider the two-point correlation function defined by

$$
\Upsilon\left(\mathbf{r}_{1}, \mathbf{r}_{2}\right) \equiv \operatorname{Tr}\left[\hat{\rho} \hat{\psi}^{\dagger}\left(\mathbf{r}_{1}\right) \hat{\psi}\left(\mathbf{r}_{2}\right)\right] .
$$

The system is said to possess the off-diagonal long-range order (ODLRO) if 33 35]

$$
\lim _{\left|\mathbf{r}_{1}-\mathbf{r}_{2}\right| \rightarrow \infty} \Upsilon\left(\mathbf{r}_{1}, \mathbf{r}_{2}\right) \neq 0 .
$$

This limiting value cannot be finite without the condensation of a macroscopic number of bosons. (Without the condensation, we simply has $\lim _{\left|\mathbf{r}_{1}-\mathbf{r}_{2}\right| \rightarrow \infty} \Upsilon\left(\mathbf{r}_{1}, \mathbf{r}_{2}\right)=0$ for the ground state and for any finite excitations.) In this sense, Eq. (165) is a criterion of the condensation.

If the system possesses the ODLRO, it is customary to define the order parameter $\Xi$ by the asymptotic form of $\Upsilon$ as

$$
\Upsilon\left(\mathbf{r}_{1}, \mathbf{r}_{2}\right) \sim \Xi^{*}\left(\mathbf{r}_{1}\right) \Xi\left(\mathbf{r}_{2}\right) .
$$


According to this definition, we obtain the same results for all of $|N, \mathbf{y}\rangle,|\xi, N, \mathbf{y}\rangle$, and $|\alpha, \mathbf{y}\rangle$, where $\xi=e^{i \phi} \sqrt{J t}$, $J t \ll N$ and $\alpha=e^{i \phi} \sqrt{N}$. Namely, using Eqs. (56) and (63), we find

$$
\lim _{\left|\mathbf{r}_{1}-\mathbf{r}_{2}\right| \rightarrow \infty} \Upsilon\left(\mathbf{r}_{1}, \mathbf{r}_{2}\right)=n_{0} \text {, hence } \Xi=\sqrt{n_{0}} e^{i \varphi},
$$

for all of these states. Therefore, neither the ODLRO nor $\Xi$ is able to distinguish between these states.

\section{B. Definition as a matrix element}

As an order parameter of the state for which $N$ is exactly fixed, Ref. 15] uses the "wavefunction of the condensate" $\Xi$, as defined by Eq. (53). It is clear that this definition is just a special case of that of the previous section. In fact, for $|N, \mathbf{y}\rangle$ Eq. (53) yields

$$
\Xi=\langle N-1, \mathbf{y}|\hat{\psi}| N, \mathbf{y}\rangle=\sqrt{n_{0}} e^{i \varphi}
$$

in agreement with Eq. (167).

\section{Definition as the expectation value of $\hat{\psi}$}

Another definition of the order parameter is the expectation value of $\hat{\psi}$, which we denote by $\Psi$;

$$
\Psi(\mathbf{r}) \equiv\langle\hat{\psi}(\mathbf{r})\rangle .
$$

According to this definition, the ground state $|N, \mathbf{y}\rangle$ of a fixed number of bosons does not have a finite order parameter;

$$
\Psi=\langle N, \mathbf{y}|\hat{\psi}(\mathbf{r})| N, \mathbf{y}\rangle=0 .
$$

This result is rather trivial because $\hat{\psi}$ alters $N$ exactly by one. On the other hand, it was sometimes conjectured in the literature [26] that the expectation value of the bare operator $\hat{a}_{0}$ might be finite $\left\langle N, \mathbf{y}\left|\hat{a}_{0}\right| N, \mathbf{y}\right\rangle \neq 0$ in the presence of many-body interactions because the number of bosons in the bare state of $\mathbf{k}=0$ fluctuates due to the many-body scatterings. However, this conjecture is wrong because by integrating Eq. (170) over $\mathbf{r}$ we obtain

$$
\left\langle N, \mathbf{y}\left|\hat{a}_{0}\right| N, \mathbf{y}\right\rangle=0 .
$$

That is, although $\hat{a}_{0}^{\dagger} \hat{a}_{0}$ fluctuates in the state $|N, \mathbf{y}\rangle$ it does not lead to a finite $\left\langle N, \mathbf{y}\left|\hat{a}_{0}\right| N, \mathbf{y}\right\rangle$.

In our gedanken experiment, $\hat{\rho}(t)$ evolves as Eq. (107), or, equivalently, as Eq. (147). For this mixed ensemble,

$$
\Psi=\operatorname{Tr}[\hat{\rho}(t) \hat{\psi}]=0 .
$$

This is the average over all elements in the mixed ensemble, and corresponds to the average over many experimental runs. On the other hand, $\Psi$ of each element, which corresponds to a possible result for a single experimental run, is different between the two expressions, Eqs. (107) and (147). That is, for each element of the mixtures, $\Psi=0$ for Eq. (107) because of Eq. (170), whereas for Eq. (147)

$$
\Psi=\left\langle e^{i \phi} \sqrt{J t}, N, \mathbf{y}|\hat{\psi}| e^{i \phi} \sqrt{J t}, N, \mathbf{y}\right\rangle
$$

can be finite. As discussed in sections $\mathrm{VD}$ and $\mathrm{VE}$, Eq. (147) is the form in which each element of the mixture has the smallest value of the phase fluctuation. This indicates that each element of the mixture possesses the most definite (non-fluctuating) value of $\Psi$ when we take the representation (147), among many representations of the same $\hat{\rho}(t)$. We are most interested in this case, because $\Psi$ is usually taken as a macroscopic order parameter, which has a definite value obeying the Ginzburg-Landau equation. 


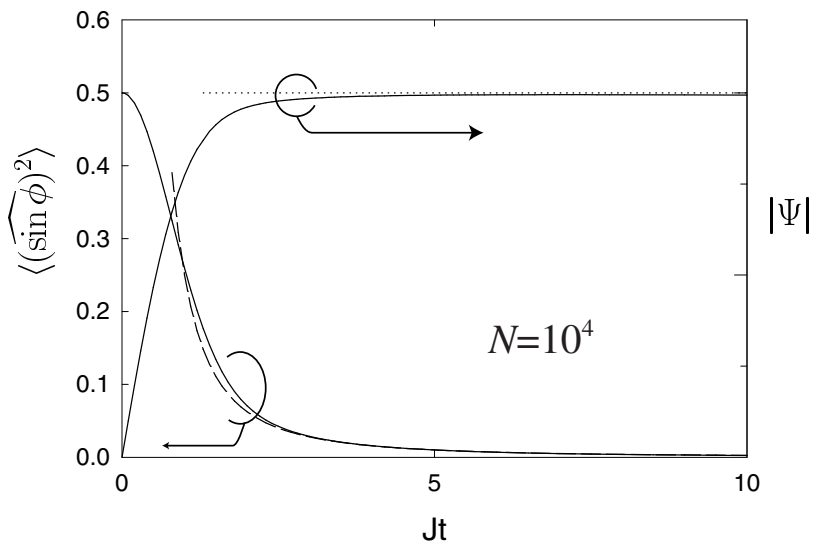

FIG. 3. Left scale: $\left\langle(\hat{\sin } \phi)^{2}\right\rangle$ of the $\phi=0$ element of Eq. (147). The dashed line represents $1 /(4 J t)$. Right scale: $|\Psi|$ defined by Eq. 173$)$. The dotted line denotes $|\Psi|=\sqrt{n_{0}}$. Both $\left\langle(\hat{\sin } \phi)^{2}\right\rangle$ and $|\Psi|$ are plotted against $J t$, the number of escaped bosons.

Figure 3 plots $|\Psi|$ of Eq. (173) as a function of $J t$ (= the average number of escaped bosons). We find that $|\Psi|$ grows very rapidly, until it attains a constant value [36],

$$
\Psi=\left\langle e^{i \phi} \sqrt{J t}, N, \mathbf{y}|\hat{\psi}| e^{i \phi} \sqrt{J t}, N, \mathbf{y}\right\rangle \rightarrow e^{i(\phi+\varphi)} \sqrt{n_{0}} \quad \text { for } J t \gtrsim 2 .
$$

This value equals $\Psi$ of $|\alpha, \mathbf{y}\rangle$ with $\alpha=e^{i \phi} \sqrt{N}$;

$$
\Psi=\langle\alpha, \mathbf{y}|\hat{\psi}| \alpha, \mathbf{y}\rangle=e^{i \varphi} \sqrt{\frac{n_{0}}{n V}} \alpha=e^{i(\phi+\varphi)} \sqrt{n_{0}}
$$

Note here that $|\Psi|$ of $|\alpha, \mathbf{y}\rangle$ is renormalized by the factor $\sqrt{|Z|}=\sqrt{n_{0} / n}$ because of the many-body interactions.

We have also plotted $\left\langle(\hat{\sin } \phi)^{2}\right\rangle$ of the $\phi=0$ element of Eq. (147) in Fig. 3. This is a measure of the phase fluctuation of the $\phi=0$ element. Because of the rotational symmetry with respect to $\phi$, we can regard it as a measure of the phase fluctuation of every element. We find that $\left\langle(\hat{\sin } \phi)^{2}\right\rangle$ decreases rapidly as $J t$ is increased, until it behaves as

$$
\left\langle(\hat{\sin } \phi)^{2}\right\rangle \approx 1 /(4 J t)
$$

for $J t \gtrsim 3$. Therefore, after the leakage of only two or three bosons, $\left|e^{i \phi} \sqrt{J t}, N, \mathbf{y}\right\rangle$ acquires the full, stable and definite (non-fluctuating) values of $\Psi$ and $\phi$, and the gauge symmetry is broken in this sense. One might expect that $\left\langle\delta N^{2}\right\rangle$ of the order of $\langle N\rangle$ would be necessary to achieve such stable $\Psi$ and $\phi$ because $\left\langle\delta N^{2}\right\rangle=\langle N\rangle$ for a CSIB. Our result shows that this expectation is wrong, because $\Psi$ and $\phi$ already become stable when $J t \sim 2$, for which $\left\langle\delta N^{2}\right\rangle=J t \ll\langle N\rangle$. Practically, it seems rather difficult to fix $N$ to such high accuracy that $\delta N \lesssim 2$. In such a case, $\delta N$ would be larger than 2 from the beginning, and each element of the mixture has the full and stable values of $\Psi$ and $\phi$ from the beginning.

We finally make a remark on the evolution at later times, whereas we have only considered the early time stage for which $J t \ll N$. It is clear that the system eventually approaches the equilibrium state. However, a question is; what is the state after the early stage, but before the system reaches the equilibrium? It is expected that the state would be some coherent state. We can show that this is indeed the case [29]: as $t \rightarrow \infty, \hat{\rho}$ eventually approaches the PRM of $|\alpha, \mathbf{y}\rangle$, in which $|\alpha|^{2}=\langle N(t)\rangle(<N)$ [36] and y is given by Eqs. (22), (23) and (33) with $n=\langle N(t)\rangle / V$ [36]. To show this, we must extend the theory of section IV. This is beyond the scope of this paper, and thus will be described elsewhere [29].

The summary of the present paper has been given in section 1 .

\section{ACKNOWLEDGMENTS}

Helpful discussions with M. Ueda, K. Fujikawa, H. Fukuyama, T. Kimura and T. Minoguchi are acknowledged. The authors also thank M. D. Girardeau for informing them of Ref. [10]. 
* $\quad$ Electronic address: shmz@ASone.c.u-tokyo.ac.jp

** Electronic address: inoue@ASone.c.u-tokyo.ac.jp

[1] For reviews, see, e.g., papers in A. Griffin et al. (eds.), Bose-Einstein Condensation, Cambridge, New York, 1995.

[2] P. Sokol, p. 51 of Ref. [1].

[3] E. Fortin, S. Fafard, and A. Mysyrowicz, Phys. Rev. Lett. 70, 3861 (1993); J. P. Wolfe, J. L. Lin and D. W. Snoke, p. 281 of Ref. [1]; L.V. Butov et al., ibid, 73, 304 (1994)

[4] M. H. Anderson et al:: Science 269 (1995) 198.

[5] C. C. Bradley et al.: Phys. Rev. Lett. 75 (1995) 1687.

[6] K. B. Davis et al.: Phys. Rev. Lett. 75 (1995) 3969.

[7] P. Nozières, p. 15 of Ref. [1].

[8] V. N. Popov, Functional integrals and collective excitations, (Cambridge, New York, 1987).

[9] E. G. Syskakis, F. Pobell and H. Ullmaier, Phys. Rev. Lett. 55 (1985) 2964.

[10] M. Girardeau and R. Arnowitt, Phys. Rev. 113, 755 (1959); M. D. Girardeau, Phys. Rev. A58, 775 (1998).

[11] J. Javanainen and S. M. Yoo, Phys. Rev. Lett. 76 (1996) 161.

[12] M. Naraschewski et al., Phys. Rev. A 54 (1996) 2185.

[13] Y. Castin and J. Dalibard, Phys. Rev. A 55 (1997) 4330.

[14] N. Goldenfeld, Lectures on Phase Transitions and the Renormalization Group (Addison-Wesley, New York, 1992).

[15] E.M. Lifshitz and L.P. Pitaevskii, Statistical Physics Part 2 (Pergamon, New York, 1980), sec. 26.

[16] Throughout this paper, we use $\sqrt{ }$ to denote the positive square root.

[17] C.W. Gardiner, Phys. Rev. A 56 (1997) 1414.

[18] Y. Castin and R. Dum, Phys. Rev. A 57 (1998) 3008.

[19] A.J. Leggett, p. 452 of Ref. [1].

[20] When we talk about the phase, we of course mean the phase relative to some reference.

[21] Actually, when $V$ is finite, we can easily show that $|0, \mathbf{y}\rangle=|0\rangle$. This equality, however, becomes meaningless when $V \rightarrow \infty$ and/or when $\hat{a}_{0}$ were replaced with a c-number. Anyway,we do not use this equality in the present paper.

[22] We can add its conjugate (creation operator) if we want to make the "coordinate" hermitian.

[23] J. Ruostekoski and D. F. Walls, Phys. Rev. A 58 (1998) R50.

[24] Since $J_{c r}$ strongly depends on the structures of the box and the hole or walls, $J_{c r}$ should be determined experimentally.

[25] See, e.g., L. Mandel and E. Wolf, Optical Coherence and Quantum Optics (Cambridge Univ. Press, 1995).

[26] See, e.g., D. Forster, Hydrodynamic Fluctuations, Broken Symmetry, and Correlation Functions (Benjamin, London, 1975), section 10.3 .

[27] Similar double pictures are well-known for free bosons, as the equivalence of the Poissonian mixture of number states and the phase-randomized mixture of coherent states 11 13].

[28] The simplest example of the double (or more) picture is a mixed state of a single spin. If the quantum state of a single spin-1/2 system is either the up-spin state $|+z\rangle$ or the down-spin state $|-z\rangle$, and if we have no information on which state is realized [in other words, the $z$ component of the spin is completely undefined], then the density matrix is,

$$
\hat{\rho}=\frac{1}{2}|+z\rangle\left\langle+z\left|+\frac{1}{2}\right|-z\right\rangle\langle-z| .
$$

On the other hand, if the direction of the spin is either in the $+x$ direction $|+x\rangle$ or in the $-x$ direction $|-x\rangle$, and if we have no information on which state is realized, then the density matrix is,

$$
\hat{\rho}=\frac{1}{2}|+x\rangle\left\langle+x\left|+\frac{1}{2}\right|-x\right\rangle\langle-x| .
$$

It is easy to verify that Eqs. (177) and (178) are identical. Therefore, the same density matrix can be interpreted in two ways; the $z$ component of the spin is definite, but unknown [Eq. (177)]; the $x$ component of the spin is definite, but unknown [Eq. (178)]. Moreover, $\hat{\rho}$ has many other representations, e.g.,

$$
\hat{\rho}=\frac{1}{2}|+y\rangle\left\langle+y\left|+\frac{1}{2}\right|-y\right\rangle\langle-y| .
$$

As demonstrated by this simple example, a mixed state of a quantum system generally has many representations, hence has many interpretations or pictures. [This is a result of the superposition principle.] Some picture is convenient depending on the physical situation. For example, Eq. (178) is most convenient to analyze the case where the $x$ component of the spin is measured.

[29] A. Shimizu et al., unpublished. 
[30] See, e.g., A. Shimizu and K. Fujita, Quantum Control and Measurement [H. Ezawa and Y. Murayama, eds., North-Holland, Amsterdam, 1993] p. 191. (quant-ph/9804026)

[31] Since the pre-measurement state [Eq. (107), or, equivalently, Eq. (147)] is a mixed state, the post-measurement state is also a mixed state if the measurement error is finite: the post-measurement state can be pure only after an error-less measurement. This may be understood by considering the measurement error and the post-measurement state as functions of the coupling strength $G$ between the measured system and the measuring apparatus: As $G$ is decreased, the measurement error increases, whereas the backaction on the measured system decreases. In the limit of $G \rightarrow 0$, the measurement error reaches $100 \%$ (i.e., no information can be obtained by such a silly measurement) and the density operator of the measured system does not change at all (hence remains a mixed state). As $G$ is increased, on the other hand, the measurement error decreases, whereas the post-measurement state approaches a pure state.

[32] M. R. Andrews et al., Science 275 (1997) 637. This type of experiment is approximately of the first kind if the leakage fluxes are small enough.

[33] O. Penrose and L. Onsager, Phys. Rev. 104 (1956) 413.

[34] C.N. Yang, Rev. Mod. Phys. 34, 694 (1962).

[35] Precisely speaking, since we consider the case of finite volume $V$, Eqs. (165) and (167) should be expressed as the following asymptotic behaviors as $\left|\mathbf{r}_{1}-\mathbf{r}_{2}\right| \sim V^{1 / 3}$

$$
\begin{aligned}
& \Upsilon\left(\mathbf{r}_{1}, \mathbf{r}_{2}\right) \sim C V^{0} \quad(C \neq 0), \\
& \Upsilon\left(\mathbf{r}_{1}, \mathbf{r}_{2}\right) \sim n_{0} V^{0} .
\end{aligned}
$$

[36] This value decreases gradually because $\langle N(t)\rangle\left(\propto n_{0}\right)$ decreases with time. 